\title{
FUNCIONES DE CONFIABILIDAD Y DESEMPEÑO SÍSMICO DE CORTINAS DE CONCRETO
}

\author{
Darío Espinoza Figueroa $^{(1)}$ y Luis Esteva Maraboto ${ }^{(1)}$
}

\begin{abstract}
RESUMEN
En este trabajo se presenta la aplicación de los principios de confiabilidad estructural para la estimación de la probabilidad de falla en cortinas de presas construidas con concreto. Se establecieron las funciones de estado límite para el agrietamiento en el cuerpo de la cortina y el deslizamiento de su base con respecto a la cimentación. Se utilizó el concepto del índice de reducción de rigidez secante presentado por Esteva et al. (2011) como una medida de la degradación de rigidez global de la cortina por la presencia de agrietamientos, a través de los resultados de un modelo bidimensional de elementos finitos (MEF2D), considerando un comportamiento no lineal en el concreto. La posibilidad del deslizamiento fue evaluada a través de los resultados del modelo MEF2D y a partir de un modelo de Cuerpo Rígido (MCR), incluyendo la variación espacial de la cohesión y el ángulo de fricción en la zona de contacto de la base de la cortina con la cimentación. Para obtener las intensidades sísmicas de falla se aplicó el concepto del análisis dinámico incremental (IDA), escalando los registros sísmicos en términos de intensidad y contenido de frecuencias, mediante el método híbrido de simulación de historias de movimiento del terreno propuesto por Ismael y Esteva (2006). Finalmente se presenta la comparación de las funciones de confiabilidad y las curvas de fragilidad de los modos de falla considerados.
\end{abstract}

Palabras clave: intensidad sísmica de falla; presas de concreto; funciones de confiabilidad

\section{RELIABILITY FUNCTIONS AND SEISMIC PERFORMANCE OF CONCRETE DAMS}

\begin{abstract}
In this paper, the application of the principles of structural reliability for the estimation of the probability of failure in concrete dams is presented. Limit state functions were established for cracking in the body of the dam and the sliding of its base with respect to the foundation. The concept of the secant stiffness reduction index presented by Esteva et al. (2011) was used as a measure of the global stiffness degradation of the dam due to the presence of cracking. The structural response and the state of cracking in the body of the dam were obtained from a two-dimensional model of finite elements (MEF2D), considering a non-linear behavior in the concrete. The sliding was evaluated through the results of the MEF2D model and from a Rigid Body (MCR) model, considering the spatial variation of cohesion and the friction angle in the contact zone of the base. The seismic intensities
\end{abstract}

Artículo recibido el 02 de noviembre de 2020 y aprobado para su publicación el 10 de diciembre de 2021. Se aceptarán comentarios y/o discusiones hasta cinco meses después de su publicación.

(1) Instituto de Ingeniería, Universidad Nacional Autónoma de México, Coyoacán, C.P. 04510, México, D.F. espinfig@hotmail.com; lestevam@iingen.unam.mx

DOI: $10.18867 /$ ris. 106.579 
leading to collapse (failure intensity) were obtained through an incremental dynamic analysis (IDA), scaling the seismic records not only in terms of intensity but also in frequency content, using the hybrid earthquake simulation method proposed by Ismael and Esteva (2006). Finally, the comparison of the reliability functions and the fragility curves of the failure modes is presented.

Keywords: seismic failure intensity; concrete dams; reliability functions

\section{INTRODUCCIÓN}

Las presas que se encuentran actualmente en operación enfrentan diversos factores que incrementan la posibilidad de colapso o mal funcionamiento, destacando los siguientes:

- Desde el punto de vista hidrológico, el cambio en el uso de suelo en la cuenca aportadora, la pérdida de cobertura vegetal y el crecimiento urbano provoca un aumento en el coeficiente de escurrimiento con el consecuente aumento en los caudales de entrada y niveles en las presas. Adicionalmente, el azolvamiento producido por los sedimentos aportados por la cuenca ya sea de forma natural o por la pérdida de cobertura vegetal, ha provocado que varias presas experimenten empujes más grandes que los previstos cuando los embalses se encuentran completamente azolvados.

- Desde el punto de vista estructural, el envejecimiento o degradación de los materiales de la cortina después de un largo tiempo (50 años, por ejemplo) causado por agentes ambientales o por historias de carga antecedentes como movimientos sísmicos, desbordamientos, etc., generan una condición de integridad estructural distinta a la que se encontraba durante el inicio de operación de la presa.

Las consecuencias producidas por el colapso de una cortina varían con el tiempo, pues están relacionadas con el desarrollo y ordenamiento de los asentamientos humanos en las zonas de inundación aguas abajo de la presa, es decir, con el aumento poblacional hay mayor exposición a las inundaciones. Todo lo anterior justifica que las presas existentes sean monitoreadas y evaluadas continuamente.

Para decidir si una presa existente guarda condiciones de seguridad satisfactorias, desde el punto de vista estructural pueden desarrollarse evaluaciones estáticas y pseudoestáticas con el fin de determinar el factor de seguridad de la estructura ante distintas condiciones de carga y escenarios de falla, considerando un modelo de cuerpo rígido. Para las cortinas tipo gravedad, de concreto o mampostería, se determina el factor de seguridad ante volteo, deslizamiento y esfuerzos excesivos en el cuerpo de la cortina. Estos análisis son considerados como "aceptación o rechazo" (Membrilla et al., 2005) pues si el factor de seguridad de la cortina es mayor que el establecido en los manuales, reglamentos o códigos de diseño se considera que la cortina no presentará falla y, en caso contrario, se declara como insegura.

Existen agencias internacionales cuyo estado del arte en términos de seguridad de presas no solo incluyen la revisión de la estabilidad de las cortinas bajo el criterio de aceptación o rechazo, sino también la estimación de la probabilidad anual de falla y de los daños asociados como es el caso del U. S Bureau of Reclamation (USBR), el U.S Army Corps of Engineers (USACE) y The Australian National Committe on Large Dams (ANCOLD). A través de la probabilidad anual de falla y los daños asociados a ella se determina si el riesgo es tolerable.

En este artículo se propone una metodología para estimar la probabilidad de falla de cortinas de concreto ante el agrietamiento en su cuerpo y el deslizamiento de su base con respecto a la cimentación. Se utiliza el concepto del índice de reducción de rigidez secante presentado por Esteva et al. (2011) como una medida de la degradación de rigidez global de la cortina por la presencia de agrietamientos, a través de los 
resultados de un modelo bidimensional de elementos finitos (MEF2D), considerando un comportamiento no lineal en el concreto. La posibilidad del deslizamiento fue evaluada a través de los resultados del modelo MEF2D y a partir de un modelo de Cuerpo Rígido (MCR), incluyendo la variación espacial de la cohesión y el ángulo de fricción en la zona de contacto de la base de la cortina con la cimentación.

\section{PROCESO DE OPTIMIZACIÓN DE LAS MEDIDAS DE REDUCCIÓN DE RIESGOS SÍSMICOS EN PRESAS}

En materia de riesgos, la norma mexicana NMX-AA-175-SCFI-2015 (DOF, 2016) establece los requisitos que deben cumplir las presas nuevas y en operación en el territorio nacional para determinar su grado de riesgo o potencial de daños en la zona posible de inundación aguas abajo por la falla parcial o total de la presa. En la norma mencionada se declara que, si las consecuencias están clasificadas como medias o altas, son inaceptables las probabilidades anuales de falla mayores a 1x10-4. Para decidir si deben implementarse acciones de reducción de riesgos se recurre a la figura 1 . Si el riesgo de la presa en estudio se ubica adentro o por arriba de las franjas diagonales mostradas en esa misma figura, entonces deben justificarse y efectuarse las acciones para reducir esos riesgos.

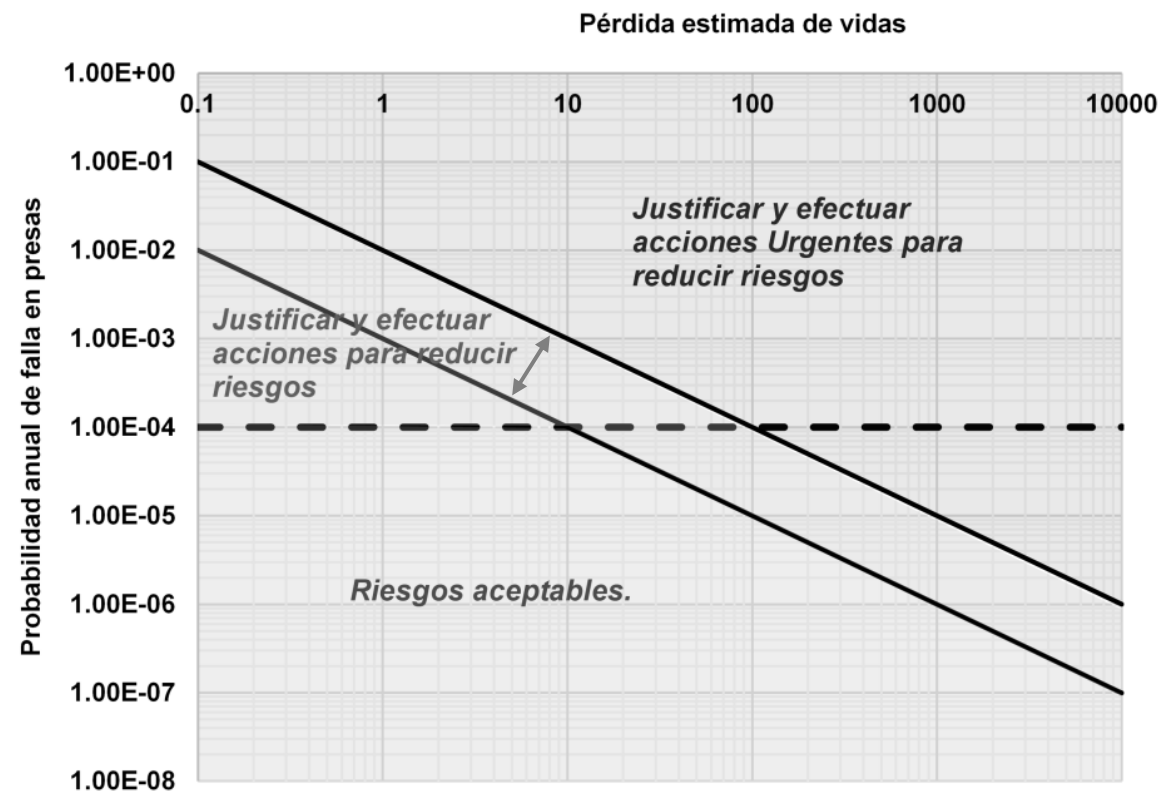

Figura 1. Criterios de aceptación de riesgos según la norma mexicana NMX-AA-175-SCFI-2015 (DOF, 2016)

La probabilidad anual de falla requerida en la figura anterior se determina individualmente para cada tipo de amenaza (hidrológica, sísmica, geológica, estructural, etc.), mientras que las consecuencias están relacionadas exclusivamente con la pérdida de vidas humanas. Cualquier medida de reducción de riesgos debe satisfacer inicialmente los criterios establecidos en la figura 1 y posteriormente considerar los aspectos económicos o de otra índole.

El planteamiento que se presenta a continuación es la base para optimizar las medidas de reducción de riesgos símicos (aunque puede utilizarse para otras amenazas), siempre y cuando las alternativas consideradas hayan satisfecho los criterios de aceptación de riesgos en términos de la pérdida de vidas humanas que se presentan en la figura 1. Para adoptar este planteamiento se requiere expresar tanto los 
costos como los beneficios de cada alternativa en términos monetarios, incluyendo el valor de la vida humana.

En el contexto de la ingeniería sísmica y dentro del marco del ciclo de vida de una obra civil, la función de utilidad (Esteva et al., 2012), $U_{t_{0}}$, está definida como la suma de los valores presentes de los beneficios y costos generados desde la construcción de la obra (tiempo $t_{0}$ ) hasta su eventual demolición o abandono (tiempo $t_{L}$ ). Para el caso especial en el que $t_{L} \rightarrow \infty$ y el sistema de interés se reconstruye inmediatamente después de cada falla, asumiendo que la función de vulnerabilidad de cada nuevo sistema es igual a la del sistema anterior, se obtiene lo siguiente:

$$
U_{t_{0}}=B-C_{0}-\mathbb{E}\left[\sum_{i=1}^{\infty} D_{i} \mathrm{e}^{-\gamma\left(t_{i}-t_{0}\right)}\right]
$$

En la ecuación anterior, $B$ es el valor presente de los beneficios esperados durante la operación de la obra, $C_{0}$ es el costo inicial de construcción, $\mathbb{E}[\cdot]$ es el valor esperado, $t_{i}$ es el tiempo desconocido de ocurrencia de un evento sísmico $i$ y $D_{i}$ es el costo asociado con el daño potencial generado por ese mismo evento $i$, incluyendo los costos de reparación y aquellos relacionados con el colapso total. Mediante la maximización de la función definida en la ecuación 1 se optimiza el desempeño esperado de la obra de interés, considerando que $t_{0}$ es el tiempo de evaluación de costos y beneficios puestos a valor presente con una tasa de descuento $\gamma$. El término $e^{-\gamma\left(t_{i}-t_{0}\right)}$ es una aproximación de $(1+\gamma)^{-\left(t_{i}-t_{0}\right)}$, que permite transformar los costos o beneficios a su valor presente siempre y cuando $\gamma \ll 1$ (Rosenblueth, 1970).

Considérese ahora el caso de una presa existente que no ha experimentado reparaciones y que se analiza en el instante $t_{E}>t_{0}$ con el fin de tomar decisiones sobre su seguridad y operación futura, suponiendo que su vida útil expira en el instante $t_{L}$. En este caso, la nueva función de utilidades $\left(U_{t_{E}}\right)$ dependerá del costo de los daños $\left(D_{j}\right)$ que pueden ocurrir en el intervalo $\left(t_{E}, t_{L}\right)$, que a su vez son función de los factores siguientes:

1. El daño inicial en la presa, presente en el tiempo de evaluación $t_{E}$, que resultó de las historias de carga y de los ataques por factores ambientales a los materiales en el periodo $t_{0} \rightarrow t_{E}$, el cual se denota por $D_{i t E}$.

2. La modificación de las funciones de vulnerabilidad sísmica mediante las estrategias de seguridad, reparación o rehabilitación $\left(R_{t E_{n}}\right)$ que se ejecuten en el tiempo de evaluación $t_{E}$ y que se describen más adelante.

3. La degradación futura de la resistencia y rigidez de los materiales, por historias de carga y/o factores ambientales, después del tiempo $t_{E}$, misma que puede denotarse como $\varrho\left(t_{j}\right)$ y que depende del tiempo.

La nueva función de utilidades, $U_{t_{E}}$, está representada esquemáticamente en la figura $2 \mathrm{y}$ puede ser expresada en términos matemáticos como sigue:

$$
U_{t_{E}}=\sum_{\mathrm{k}=1}^{N_{B F A}}\left(B_{F A k} \mid R_{t E_{n}}\right)-\mathbb{E}\left[\sum_{j=1}^{\infty}\left[D_{j} \mid D_{i t E}, R_{t E_{n}}, \varrho\left(t_{j}\right)\right] e^{-\gamma\left(t_{j}-t_{E}\right)}\right]-C_{R_{t E_{n}}}
$$

donde $N_{B F A}$ es el número de tipos de beneficios futuros esperados por el uso del agua (agrícola, público urbano, generación de energía eléctrica, etc.) expresados generalmente en forma anual, $B_{F A k}$ es el valor presente de esos beneficios futuros, $R_{t E_{n}}$ son las acciones de seguridad, reparación o rehabilitación 
ejecutadas en el instante de evaluación $t_{E}$ que se describen más adelante y cuyo costo total es $C_{R_{t E_{n}}}, D_{j}$ es el costo total de los daños asociados a cada evento sísmico $j$ con tiempo desconocido de ocurrencia $t_{j}$, incluyendo los relacionados con la falla total o parcial de las estructuras que forman parte de la presa, así como los daños incrementales aguas abajo por los caudales descargados. Tanto $D_{i t E}$ como $\varrho\left(t_{j}\right)$ se definieron anteriormente. En la ecuación 2 tanto los beneficios, $B_{F A k}$, como el costo de los daños, $D_{j}$, deben actualizarse a valor presente mediante la tasa de descuento $\gamma$.

Tomando como referencia las tendencias actuales en materia de seguridad de presas, las estrategias de seguridad, reparación o rehabilitación, $R_{t E_{n}}$, incluidas en la ecuación 2, pueden agruparse en cinco:

- $R_{t E_{1}}=$ la presa continuará sin reparaciones, operando de la misma forma o con cambios en su política de operación. En este caso $C_{R_{t E_{n}}}=0$, en la ecuación 2 .

- $R_{t E_{2}}=$ la presa se rehabilitará hasta un nivel de confiabilidad sísmica aceptable, operando de la misma forma o con cambios en su política de operación.

- $R_{t E_{3}}=$ la presa se modificará o rehabilitará hasta un nivel de confiabilidad sísmica aceptable, con cambios en el uso del agua y, por lo tanto, en los beneficios.

- $R_{t E_{4}}=$ se removerán una o varias estructuras de la presa.

- $R_{t E_{5}}=$ se removerán completamente todas las estructuras que conforman la presa, por lo que, en la ecuación $2, B_{F A k}=D_{j}=0$.

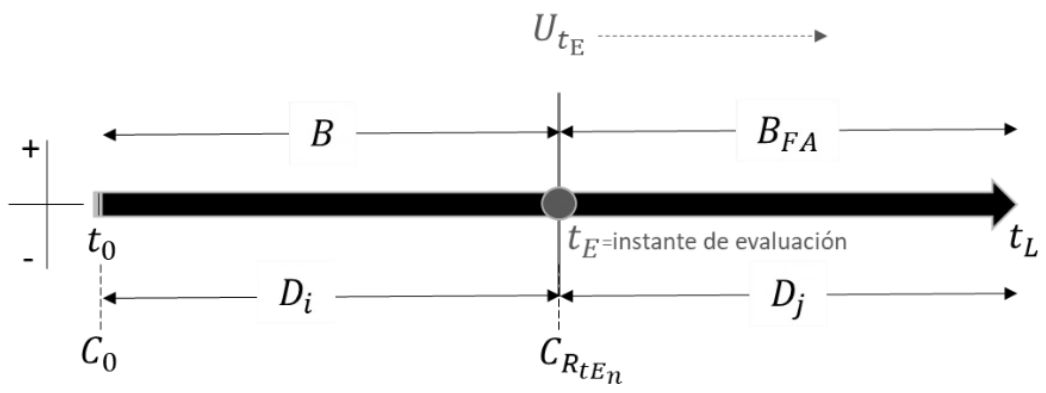

Figura 2. Representación esquemática de la línea de tiempo del ciclo de vida de una presa y de las utilidades esperadas en el instante de evaluación $t_{E}>t_{0}$

A primera vista podría pensarse que la estrategia $R_{t E_{n}}$ que genere la utilidad mayor sería la más conveniente, sin embargo, más allá de los aspectos económicos existen factores sociales, culturales, legales, ambientales y de ordenamiento territorial que pueden ser decisivos sobre mantener una presa, repararla, rehabilitarla o removerla parcial o totalmente. En la medida en que estos factores pueden convertirse en valores monetarios pueden incluirse en la función de utilidades de la ecuación 2 y, en caso contrario, evaluarse de forma separada.

\section{FUNCIONES DE VULNERABILIDAD SÍSMICA EN PRESAS}

Considerando que el costo de los daños $D_{j}$ son producidos por eventos sísmicos $j$ cuando la presa tiene un nivel fijo de agua $h$, entonces con base en Esteva et al. (2011) se puede establecer que si una presa continúa sin reparaciones después del tiempo de evaluación $t_{E}$ (estrategia $R_{t E_{1}}$ ), mismo que puede ser 
tomado como un escenario de referencia para valorar las estrategias de seguridad $R_{t E_{2}}$ a $R_{t E_{5}}$, entonces el costo de los daños $D_{j}$ puede estimarse como sigue:

$$
D_{j}=\mathbb{E}[\delta \mid y, h]=p_{F}\left(y \mid h, D_{i t E}, R_{t E_{1}}, \varrho\left(t_{j}\right)\right) \delta_{F}+\left[1-p_{F}\left(y \mid h, D_{i t E}, R_{t E_{1}}, \varrho\left(t_{j}\right)\right)\right] \delta(y, h \mid S)
$$

En la ecuación 3 el término $\mathbb{E}[\delta \mid y, h]$ se refiere a la esperanza del costo de los daños $\delta$ producidos por la falla de la presa cuando se presenta un sismo con intensidad $y$ y el embalse tiene un nivel de agua $h$, $p_{F}(y \mid h)$ es la probabilidad de falla última (colapso) de la cortina o cualquier componente de la presa cuando está sujeta al sismo con intensidad $y$ dado que el embalse tiene el nivel $h, \delta_{F}$ es el costo esperado del colapso en caso de que ocurra y $\delta(y, h \mid S)$ es el costo esperado de los daños para un sismo con intensidad $y$ y un nivel $h$ en el vaso, condicionados a la sobrevivencia de la presa. Nótese que la probabilidad de falla no depende solo de la intensidad sísmica y y del nivel del agua $h$, sino también del daño inicial, $D_{i t E}$, así como de la función de degradación de la resistencia y rigidez, $\varrho\left(t_{j}\right)$, que varía con el tiempo. Con la ecuación 3 pueden elaborarse curvas de vulnerabilidad sísmica suponiendo que tanto la intensidad sísmica ( $y$ ) como el nivel del agua $(h)$ son conocidos y las propiedades mecánicas de los materiales son variables aleatorias.

El nivel del agua $(h)$ depende de la política operativa de la presa y en algunas de ellas se establecen, entre dependencias gubernamentales, curvas guía que permiten establecer el nivel máximo permitido del embalse para cada quincena del año. Este nivel máximo es disminuido en la temporada de lluvias y aumentado durante la época de estiaje, dependiendo del régimen de escurrimientos de la cuenca de aportación. Con la consideración explícita de la curva guía en la aplicación de la ecuación 3 es posible estimar el cambio en la vulnerabilidad de la estructura por cambios en su política operativa.

Si la presa no cuenta con una política de operación definida, pero se tienen registros de los niveles del embalse, puede desarrollarse un análisis estadístico para elegir cierto nivel fijo característico del agua y realizar los análisis sísmicos correspondientes. Debe tenerse presente que la probabilidad de que ocurra un sismo de magnitud importante cuando se tienen un nivel extraordinario en el embalse es muy baja, por tanto, el nivel considerado debe estar asociado a una condición normal de operación.

Por otro lado, es importante recordar que, para algunos tipos de presas, como las de tierra y enrocamiento, la condición sísmica más desfavorable del talud aguas arriba no se obtiene siempre bajo una presa llena (MOC, 2015) por lo que la elección del nivel del agua para fines de análisis sísmico no es un asunto trivial.

Por otro lado, la función $p_{F}\left(y \mid h, D_{i t E}, R_{t E_{n}}, \varrho\left(t_{j}\right)\right)$ puede utilizarse para obtener la tasa esperada de falla que, de acuerdo con Esteva et al. (2002), se calcula mediante la expresión siguiente:

$$
v_{F}=\int_{0}^{\infty}\left|\frac{d v_{Y}(y)}{d y}\right| p_{F}\left(y \mid h, D_{i t E}, R_{t E_{n}}, \varrho\left(t_{j}\right)\right) d y
$$

En la ecuación anterior, $v_{F}$ es la tasa esperada de falla; si la tasa de excedencias de las intensidades sísmicas $v_{Y}(y)$ se expresa en términos anuales, entonces $v_{F}$ representa la tasa anual de falla. La expresión $v_{Y}(y)$ representa la curva de peligro sísmico y se obtiene conforme a la ecuación 5.

$$
v_{Y}(y)=\sum_{i=1}^{N f} \int_{M_{0}}^{M_{u}}-\frac{d \lambda(M)}{d M} P(Y>y \mid M, R) d M
$$


donde $N f$ es el número de fuentes sísmicas, $M_{0}$ y $M_{u}$ son las magnitudes mínima y máxima consideradas dentro del catálogo de eventos sísmicos de cada fuente, respectivamente, $\lambda(M)$ es la tasa de excedencias de las magnitudes y $R$ es la distancia representativa desde la fuente sísmica hasta el sitio en estudio. En adición, si se considera que el tiempo de ocurrencia $T$ entre fallas sigue un proceso de Poisson homogéneo, entonces la probabilidad anual de falla $p_{F(1 a n ̃ o)}$ se calcula mediante una función exponencial de probabilidad con parámetro $v_{F}$, de tal forma que:

$$
p_{F(1 \text { año })}=P(T \leq 1)=1-e^{-v_{F}}
$$

Para el caso específico de presas, con la ecuación anterior puede calcularse la probabilidad anual de colapso, con el fin de evaluar si las condiciones de seguridad que guarda una cortina satisfacen el riesgo tolerable especificado en la figura 1.

\section{MECANISMOS DE FALLA}

En el Manual de Capacitación de Seguridad de Presas de la Comisión Nacional del Agua (CONAGUA, 2001) se menciona que los modos principales de falla de presas rígidas ante excitaciones sísmica son la falla por esfuerzos internos, en el cuerpo de la cortina y la falla de la cimentación. En presas existentes se han reportado agrietamientos por esfuerzos excesivos de tensión en la ubicación de discontinuidades geométricas de la cortina. Estos esfuerzos de tensión son la causa principal de agrietamiento en presas de concreto. En la figura 3 se muestra un esquema con el comportamiento de una presa rígida de concreto durante una excitación sísmica.

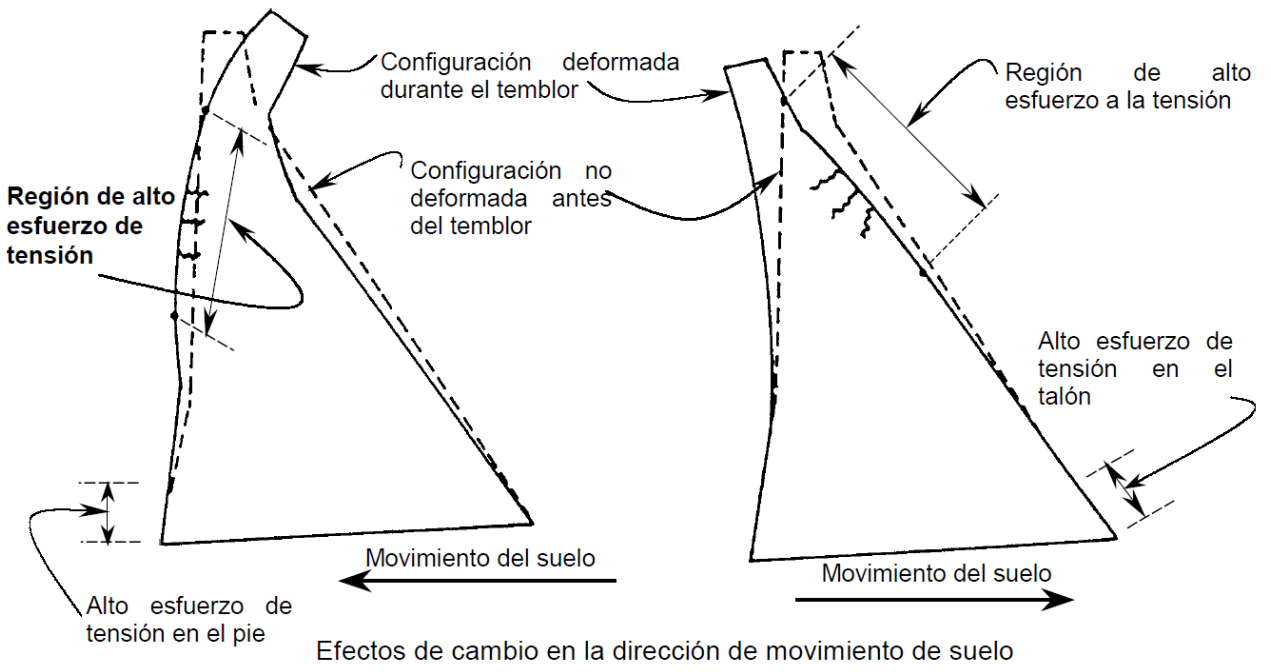

Figura 3. Comportamiento de una presa durante una excitación sísmica. Fuente: CONAGUA (2001)

Con respecto a la falla de la cimentación, importa evaluar los movimientos en fallas geológicas u otras zonas débiles de la cimentación y los empotramientos. Las deformaciones excesivas de la cimentación pueden causar agrietamiento en la cortina y producir la falla. También se considera la posibilidad de deslizamientos en planos de estratificación y zonas de debilidad de la cimentación tomando en cuenta los parámetros de resistencia post-sísmica (figura 4). 


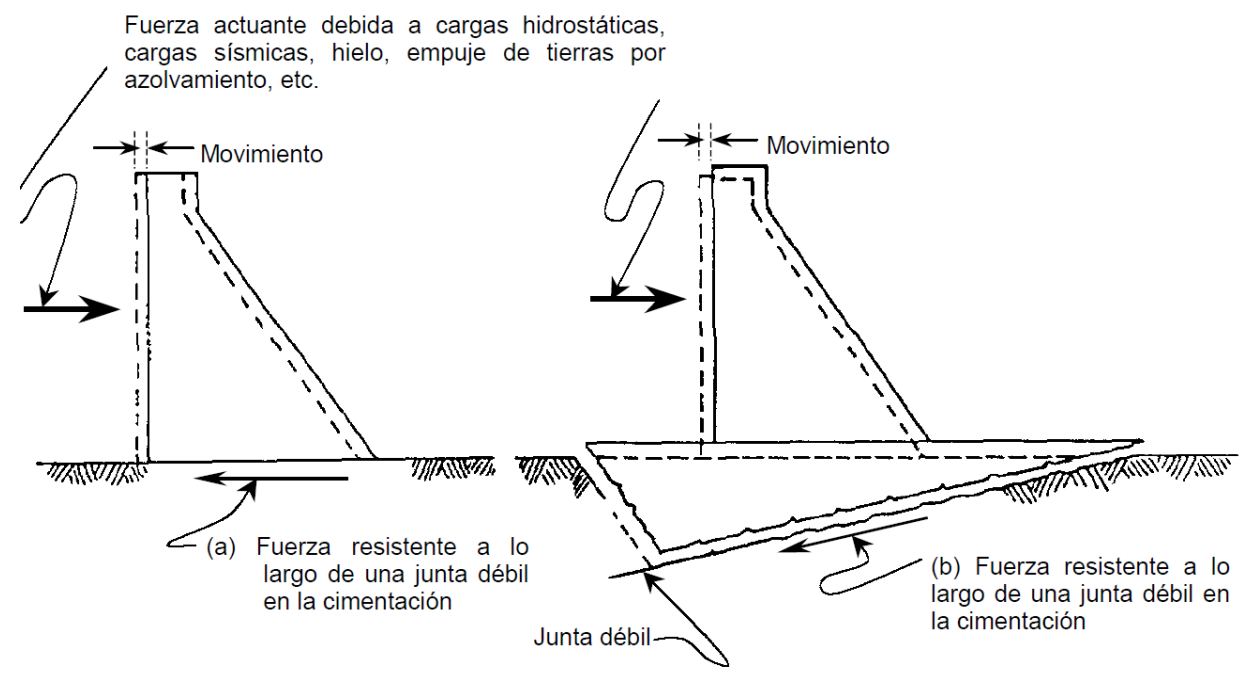

Figura 4. Representación de la falla por deslizamiento. Fuente: CONAGUA (2001)

La Comisión Internacional en Grandes Presas (ICOLD, 1995) publicó un análisis estadístico de más de 170 fallas en presas construidas en distintas partes del mundo detallando las causas que produjeron el comportamiento inadecuado en cortinas de concreto y mampostería. En específico, se reportaron fallas debido a las causas siguientes:

- Fallas debidas al comportamiento estructural: esfuerzos de tensión.

- Fallas en cimentación: Poca investigación del sitio, resistencia al corte, filtraciones, erosión interna, esfuerzos de tensión en el talón de la cortina.

- Fallas debidas al concreto: esfuerzos producidos por congelamiento y descongelamiento, alta permeabilidad del concreto, envejecimiento.

- Fallas debidas a acciones imprevistas o cuyas magnitudes fueron excepcionales: subpresión y desbordamientos.

\section{METODOLOGÍA PROPUESTA PARA LA ESTIMACIÓN DE PROBABILIDADES DE FALLA}

Se presenta la aplicación de una metodología para determinar la probabilidad de falla de cortinas de concreto y, mediante la aplicación de la ecuación 4, permitan la evaluación del riesgo tolerable conforme a la normativa mexicana, según la figura 1. Por simplicidad, en este artículo se asume que no existe un nivel inicial de daño, no se considerarán estrategias de reparación y rehabilitación estructural y no se prevé degradación de resistencia, rigidez o daño acumulado a lo largo del tiempo. Con lo anterior, los términos $D_{i t E}, R_{t E_{\mathrm{n}}}$ y $\mathrm{Q}\left(t_{j}\right)$ expresados en las ecuaciones 2 y 3 pueden ignorarse, por lo que la probabilidad de falla puede denotarse como $p_{F}(y \mid h)$.

\section{Funciones de estado límite}

Atendiendo los modos de falla reportados en la literatura se propone la evaluación de la probabilidad de falla bajo dos escenarios principales, independientes entre sí: el primero es el agrietamiento en el cuerpo de la cortina y el segundo es el deslizamiento en su base, aunque este último puede ser utilizado para evaluar el deslizamiento en cualquier plano débil de falla en la roca de cimentación. 
Sea $G_{c k}(\bar{x})$ la función de estado límite expresada en función de las variables inciertas $\bar{x}$ involucradas en cierto modelo de la cortina, entonces, el margen de seguridad para el agrietamiento puede expresarse en términos del índice de reducción de rigidez secante de la estructura $\left(I_{S S R}\right)$ presentado por Esteva et al. (2011) como sigue:

$$
G_{c k}(\bar{x})=1-I_{S S R} \sim 0
$$

donde $I_{S S R}=\frac{K_{0}-K}{K_{0}}$. El término $K_{0}$ es la rigidez tangente global inicial de la cortina obtenida a través de un análisis dinámico considerando una intensidad sísmica poco significativa (que no provoque comportamientos no lineales) y $K$ es la rigidez secante calculada con el valor máximo absoluto del desplazamiento relativo de la corona de la cortina con respecto a su base, durante la respuesta no lineal de la cortina y su cortante basal asociado, expresada mediante ciclos de histéresis. En este trabajo los ciclos de histéresis se obtuvieron mediante modelos bidimensionales utilizando el método de Elemento Finito (MEF2D) considerando un comportamiento no lineal en el concreto.

La condición de falla se expresa como $I_{S S R} \sim 1$. La confiabilidad se determina ajustando una función lognormal de probabilidad a todos los valores mínimos de la intensidad sísmica de falla $\left(Y_{F}\right)$ que satisfacen esa condición. Haciendo $Z_{F}=\ln Y_{F}$ la función de confiabilidad puede expresarse como (Esteva et al., 2011):

$$
\beta(y)=\frac{E\left(Z_{F}\right)-\ln y}{\sigma\left(Z_{F}\right)}
$$

En la ecuación anterior $E(\cdot)$ y $\sigma(\cdot)$ representan el valor esperado y la desviación estándar, respectivamente. Para el deslizamiento de la cortina se consideran dos criterios para establecer la función de estado límite. El primero está basado en Bernier et al. (2016) quienes mencionaron los niveles de daños relacionados con la magnitud del deslizamiento en la base de una cortina de concreto, basado en el juicio de expertos. Estos investigadores señalaron que un deslizamiento de $150 \mathrm{~mm}$ implicaría un nivel de daño severo que causaría movimientos diferenciales inaceptables en relación con los monolitos adyacentes y podría, eventualmente, causar el colapso total y, por consiguiente, la liberación descontrolada del agua de la presa. Bajo estas consideraciones, la función de estado límite se expresa con la ecuación siguiente:

$$
G_{s l 1}(\bar{x})=150 \mathrm{~mm}-\mathrm{S}_{l b_{-} F E M 2 D} \leq 0
$$

donde $S_{l b}$ es el deslizamiento en la base de la cortina obtenido del análisis MEF2D.

El segundo criterio para establecer la función de estado límite para el deslizamiento de la cortina es mediante la consideración de un modelo de cuerpo rígido (MCR), más simple que el modelo MEF2D, pero usado ampliamente en la práctica profesional durante las etapas de diseño preliminar, basado en un modelo de falla tipo Mohr-Coulomb. Se incluye una discretización de la base de la cortina, para considerar variaciones en la cohesión y el ángulo de fricción conforme a los hallazgos de Westberg y Johansson (2013) quienes coordinaron la perforación y obtención de 8 núcleos del contacto cortina-cimentación en la presa Laxeden, Suecia. De las 8 muestras, 5 resultaron intactas (con cohesión) y 3 mostraron el contacto concretoroca con agrietamiento (sin cohesión). En este caso, la función de estado límite para el deslizamiento $G_{s l 2}(\bar{x})$ queda expresado, partiendo de un modelo Mohr-Coulomb, como sigue:

$$
G_{s l 2}(\bar{x})=\sum_{i=1}^{k} \mathrm{c}_{i} \mathrm{~A}_{i}+\mathrm{N}_{i} \mu_{i}-\sum F_{h} \leq 0
$$


En la ecuación anterior $k$ representa el número total de discretizaciones consideradas en la base de la cortina, $c_{i}$ es la cohesión, $A_{i}$ es el área de la discretización, $N_{i}$ es la fuerza normal actuando en la discretización, $\mu_{i}$ es el coeficiente de fricción calculado como $\mu_{i}=\tan \left(\phi_{i}\right)$, siendo $\phi_{i}$ el ángulo de fricción y el término $\sum F_{h}$ indica la sumatoria de todas las fuerzas horizontales actuando en la cortina (presión hidrostática, presión de azolves, fuerza inercial horizontal, presión hidrodinámica y presión dinámica de azolves); el subíndice $i$ indica la discretización correspondiente. La congruencia con las observaciones de Westberg y Johansson (2013) se dará si se considera que cuando más el 60\% de los tramos resultantes de la discretización de la base de la cortina tiene cohesión y fricción y el 30\% restante únicamente fricción.

\section{Modelos de comportamiento estructural}

La geometría de la cortina y las solicitaciones impuestas se muestran en la figura 5. Se consideró una altura de cortina de 40 metros y un nivel fijo en el embalse $(h)$ a 36 metros sobre el nivel del fondo del cauce y sus respectivas masas adheridas para representar los efectos hidrodinámicos. Se supuso una eficiencia del 55\% en el sistema de drenaje y un espesor de 10 metros de azolves. La sección geométrica de la cortina fue diseñada satisfaciendo los factores de seguridad ante volteo, deslizamiento y esfuerzos excesivos en su base, conforme a las recomendaciones del Manual de Obras Civiles de la Comisión Federal de Electricidad (MOC, 2015). Se utilizó como coeficiente sísmico horizontal $k_{h}=a_{0} /\left(1+2 a_{0}\right)$ actuando en la dirección aguas abajo, con $a_{0}$ definida como la aceleración máxima en roca (PGA, por sus siglas en inglés), asociada al nivel de intensidad sísmica de diseño (probabilidad anual de excedencias 1/10,000). Adicionalmente se consideró un coeficiente sísmico vertical tomado como $k_{v}=2 k_{h} / 3$.

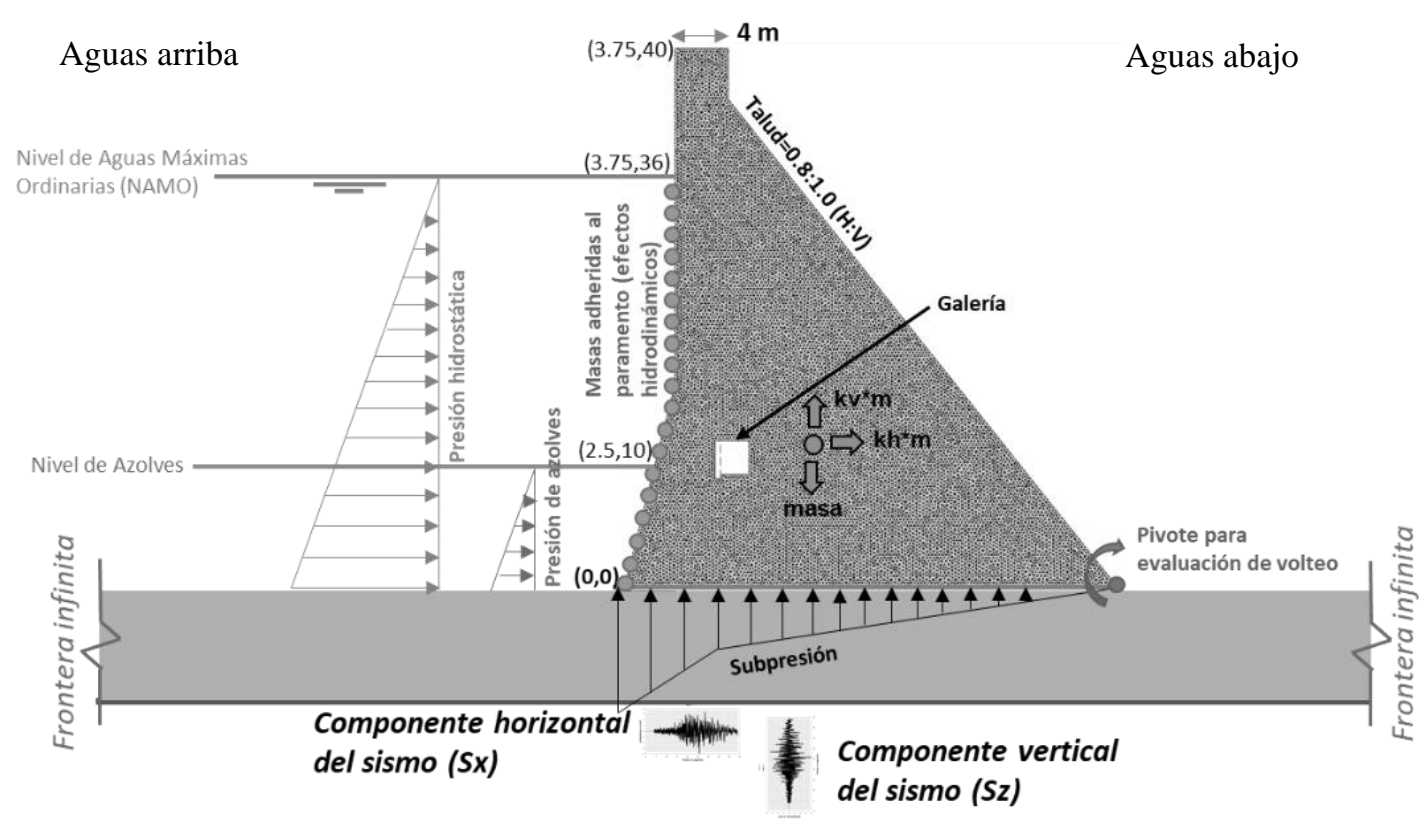

Figura 5. Características de la cortina analizada en este estudio

La evaluación de las funciones de estado límite fue desarrollada mediante dos modelos de comportamiento estructural: MEF2D y MCR. Con el modelo MEF2D se desarrolló un análisis dinámico paso a paso en el dominio del tiempo a través del software ABAQUS, considerando un comportamiento no lineal en el concreto mediante un modelo continuo de daño por falla a tensión y compresión que se inicia cuando los esfuerzos principales alcanzan las resistencias ante ambas condiciones. Durante el comportamiento no lineal a tensión se asumió el criterio de agrietamiento mediante la energía de fractura 
propuesto por Hillerborg's en 1976, considerando una curva bilineal de ablandamiento por deformación. Para compresión, se utilizó la parábola de Hognestad para representar la curva esfuerzo-deformación en el rango no lineal. Las propiedades mecánicas y de resistencia de los materiales de la cortina para la construcción de las relaciones constitutivas mencionadas anteriormente se presentan en la tabla 1, descrita en los apartados siguientes. Las historias de aceleración utilizadas en los análisis tuvieron un tiempo total de 80 segundos.

Por otro lado, la base de la cortina fue discretizada en 10 secciones de las que 6 incluyeron cohesión y fricción $(\mathrm{C}+\mathrm{F})$ en el contacto con la cimentación y 4 incluyeron únicamente fricción $(\mathrm{F})$, tomando como base las observaciones efectuadas por Westberg y Johansson (2013). Esta consideración fue ejecutada tanto en el modelo MEF2D como en el modelo MCR (ver figura 6).

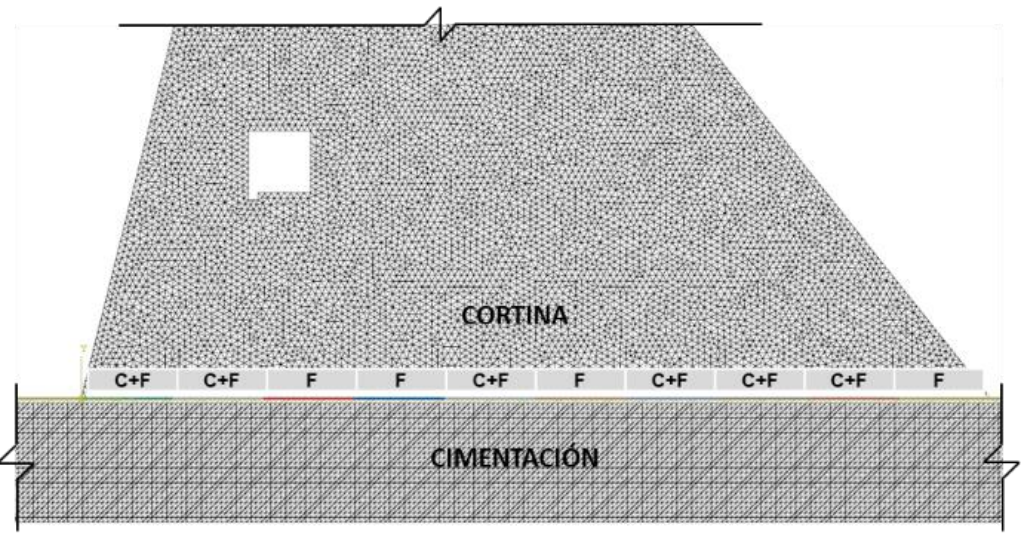

Figura 6. Ejemplo de discretización de la base de la cortina en el modelo de comportamiento estructural

\section{Caracterización de la amenaza sísmica}

La estimación del desempeño estructural de una cortina rígida, de acuerdo con las funciones de estado límite descritas anteriormente, requiere el uso de historias de aceleración en el tiempo (acelerogramas) relacionadas con el movimiento sísmico. Los valores numéricos de estos acelerogramas pueden obtenerse de una base de datos de registros reales o generarse sintéticamente. Es necesario además que los acelerogramas estén asociados a un nivel de intensidad sísmica, pero en varias ocasiones las bases de datos no cuentan con registros suficientes para relacionarlos con el nivel de intensidad de interés. Por lo anterior, es común emplear técnicas para generar acelerogramas sintéticos mediante el escalamiento de registros reales y llevarlos hasta el nivel de intensidad sísmica requerida.

A diferencia del método de escalamiento de registros sísmicos a través de un escalar descrito por Vamvatsikos y Cornell (2002), en este trabajo se considera la aplicación del método híbrido de simulación de historias del movimiento del terreno propuesto por Ismael y Esteva (2006) que aprovecha las ventajas las funciones generalizadas de atenuación (Alamilla et al., 2001) y las funciones de Green empíricas (Ordaz et al., 1995 ). Con lo anterior se pretende que los registros sísmicos sean escalados tanto en términos de intensidad sísmica como en contenido de frecuencias. Para lograr lo anterior, se procesaron 455 registros sísmicos de 7 estaciones ubicadas en el estado de Chiapas, México, que ocurrieron entre los años 1976 a 2016. Los registros sísmicos están contenidos en la base de datos de registros acelerográficos de la red sísmica del Instituto de Ingeniería de la UNAM.

En la figura 7 se muestra la ubicación de los epicentros, las estaciones acelerográficas y un sitio elegido para caracterizar la amenaza sísmica mediante el método híbrido mencionado anteriormente. Con los datos de magnitud, distancia y aceleraciones de los 455 registros sísmicos y la integración numérica de 
la ecuación 5 se obtuvo la tasa de excedencias de las intensidades sísmicas $v_{Y}(y)$ en el sitio de interés (ver figura 8).

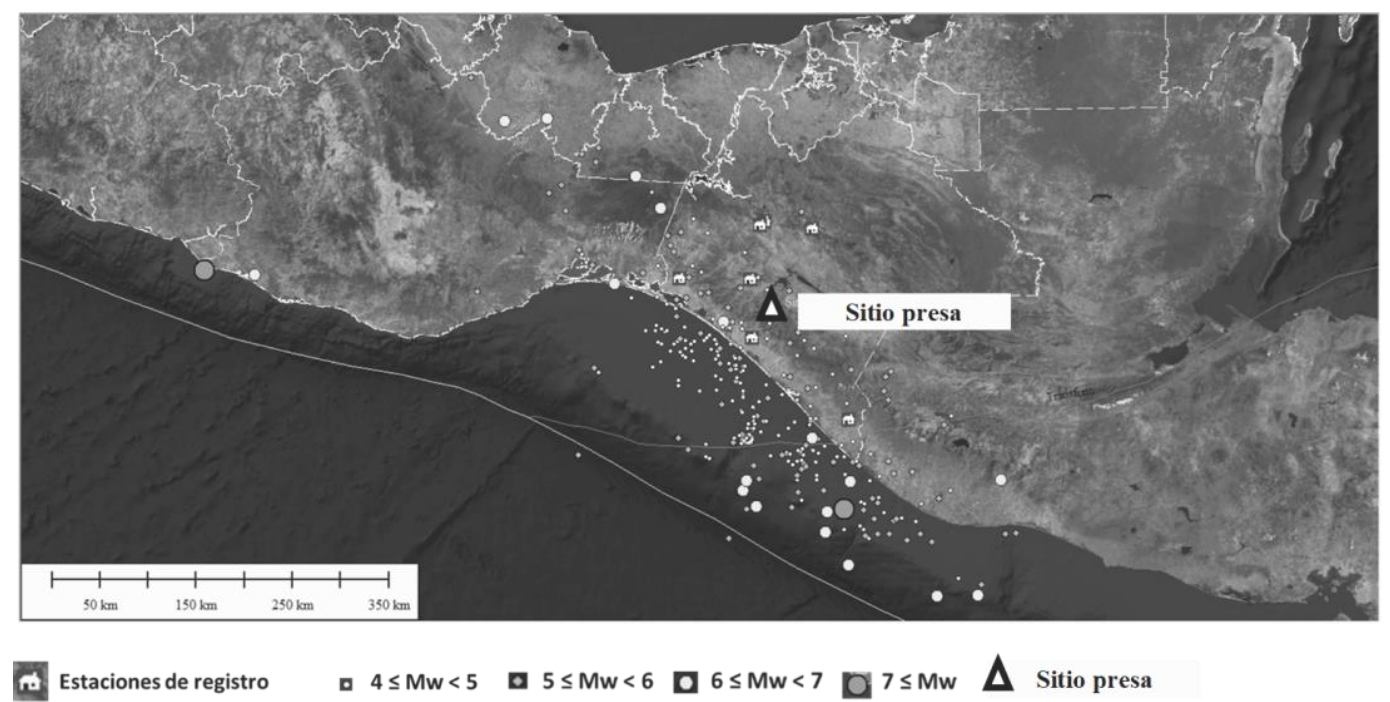

Figura 7. Epicentros y estaciones acelerográficas utilizadas para caracterizar la amenaza sísmica. Imagen de Global Mapper

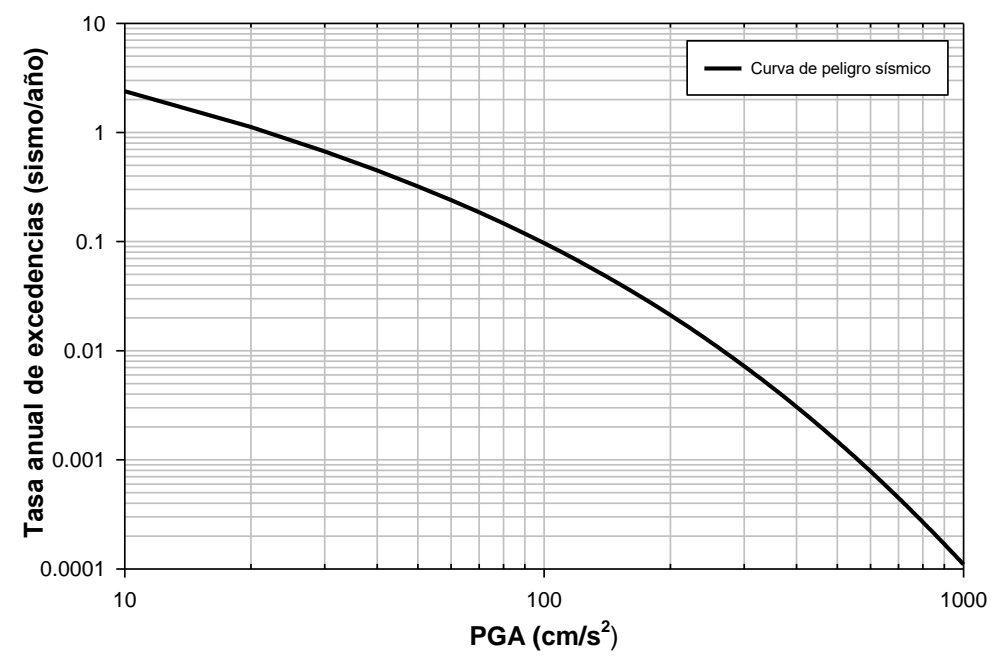

Figura 8. Tasa anual de excedencias de PGA como resultado del procesamiento de los 455 registros sísmicos en el estado de Chiapas

De acuerdo con el procedimiento propuesto por Alamilla et al. (2001), se estimaron la magnitud $M$ y distancia $R$ más probables del evento que generaría la intensidad asociada a cierta probabilidad anual de excedencias, partiendo de la función de densidad de probabilidad conjunta de $M$ y $R$ para un valor dado de la intensidad sísmica $Y=y$, misma que fue obtenida a partir del teorema de Bayes y que está representada en la ecuación 11.

$$
f_{M, R}(m, r \mid y)=k f_{Y}(y \mid m, r) f_{(M, R)}(m, r)
$$

En la ecuación anterior, $k$ es una constante de normalización tal que la integral de $f_{M, R}(m, r \mid y)$ sobre el dominio de valores posibles de $M$ y $R$ es igual a la unidad, $f_{Y}(y \mid m, r)$ es la función de densidad de probabilidad condicional de la intensidad sísmica $Y$ para $M=m$ y $R=r$ y $f_{(M, R)}(m, r)$ es la función de 
densidad de probabilidad conjunta de $M$ y $R$. De forma inicial, se obtuvo un valor aleatorio de $M$ condicionado a la intensidad sísmica de interés mediante la ecuación 12.

$$
f_{M}(m \mid y)=k \int f_{Y}(y \mid m, r) f_{(M, R)}(m, r) d r
$$

Para obtener la densidad de probabilidad $f_{Y}(y \mid m, r)$ se consideró la función de densidad de probabilidad del error estadístico de la función de atenuación considerada en la ecuación 5. Este error estadístico se obtiene a través de la relación $y 1 / y c$, donde $y 1$ es la aceleración observada y $y c$ la aceleración obtenida con la regresión. Esta relación se ajustó a una función de probabilidad lognormal Log[-0.0076, 0.917], como se muestra en el histograma de la figura 9.

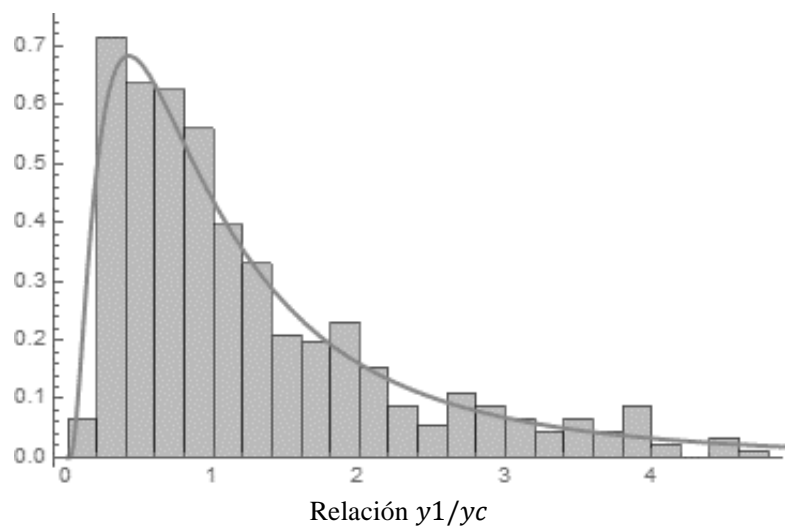

Figura 9. Función de densidad de probabilidad de la relación $y 1 / y c$

La función de densidad de probabilidad conjunta $f_{(M, R)}(m, r)$ se tomó como el producto de las probabilidades marginales $f_{M}(m) f_{R}(r)$. La función de densidad de probabilidad $f_{M}(m)$ se representa mediante la expresión dada por Alamilla et al. (2001) presentada en la ecuación 13, considerando $M o, M u$ y $\beta$ tal y como fueron definidas en la ecuación 5.

$$
f_{M}(m)=\frac{\beta e^{-\beta M}}{e^{-\beta M_{0}}-e^{-\beta M_{\mathrm{u}}}}
$$

La densidad de probabilidad de la distancia, $f_{R}(r)$ se obtuvo mediante inferencia estadística, a través del ajuste de una función de probabilidad a las distancias focales observadas, de acuerdo con el procesamiento geográfico de esas distancias al sitio de interés, para los 455 registros sísmicos mostrados en la figura 7. Para este caso, la función de probabilidad encontrada es una función lognormal con parámetros $\log [5.114,0.335]$. En la figura 10 se muestra el histograma con la función de densidad de probabilidad ajustada para $f_{R}(r)$, a partir de los datos observados.

Para cada valor supuesto de la intensidad y se simuló un valor de $M$, de acuerdo con la ecuación 12, y se simuló un valor de la distancia focal $R$, considerando la función de densidad de probabilidad de la distancia condicionada a la magnitud y la intensidad sísmica de interés, expresada matemáticamente en la ecuación 14.

$$
f_{R}(r \mid m, y)=\frac{f_{M, R}(m, r \mid y)}{f_{M}(m \mid y)}
$$




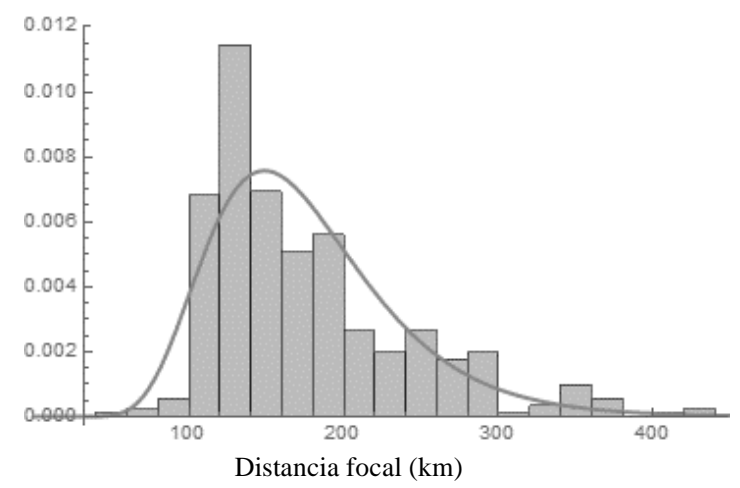

Figura 10. Función de densidad de probabilidad $f_{R}(r)$ de las distancias focales al sitio de interés, obtenida con los datos observados procesados mediante el uso de un Sistema de Información Geográfica

Considerando el procedimiento anterior se llevaron a cabo 1,000 simulaciones de la magnitud y distancia, asociados a distintos niveles de intensidad sísmica. Se escribió un código de programación en el lenguaje Wolfram-Mathematica con el fin de realizar la integración numérica del método híbrido. En la figura 11 se muestra la función de probabilidad conjunta $f_{M, R}(m, r \mid y)$ para las intensidades asociadas a probabilidades de excedencia de 1/10,000,1/3,000, 1/1,000 y 1/145. Finalmente, para la generación de los acelerogramas sintéticos incluyendo las tres direcciones ortogonales (ahmax, ahmin y avert) se utilizó el método de las funciones de Green empíricas descrito en Ordaz et al. (1995), utilizando 6 registros semilla. En la figura 12 se muestra el ejemplo de los acelerogramas sintéticos generados con el método híbrido para las tres componentes ortogonales, asociados al nivel de intensidad sísmica correspondiente a una probabilidad de excedencias del 50\% en 100 años. En última instancia, en la figura 13 se presentan los espectros de respuesta elástica de 10 acelerogramas sintéticos generados para un nivel de intensidad sísmica asociada a una probabilidad de excedencias del $50 \%$ en 100 años.
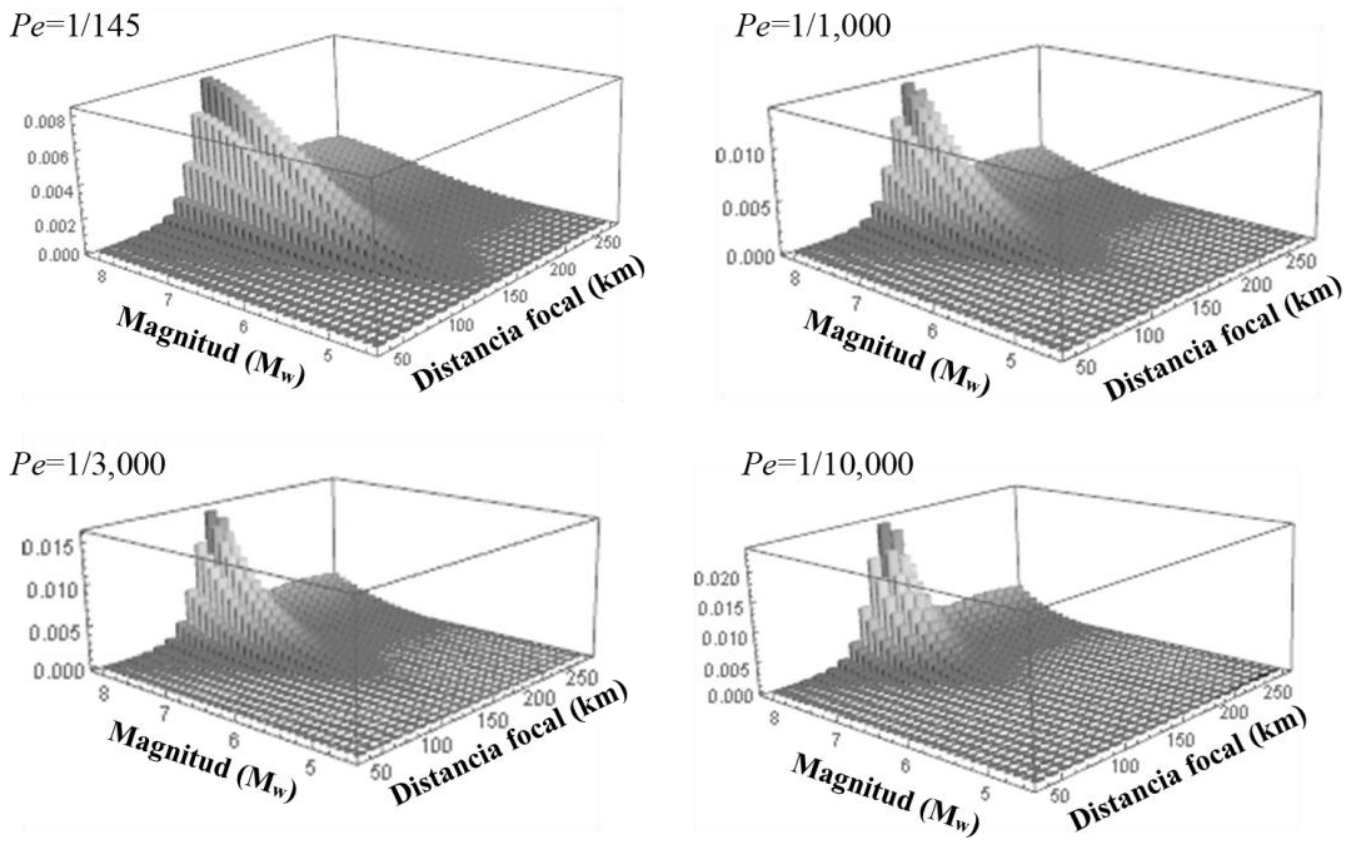

Figura 11. Función de densidad de probabilidad conjunta $f_{M, R}(m, r \mid y)$ como resultado del procesamiento de 455 sismos en el estado de Chiapas 
a)
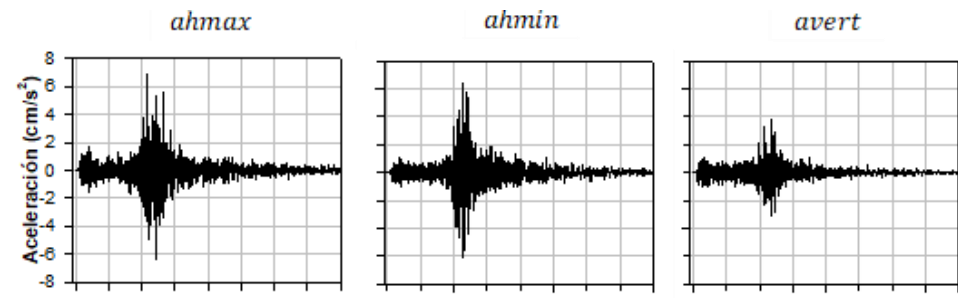

b)
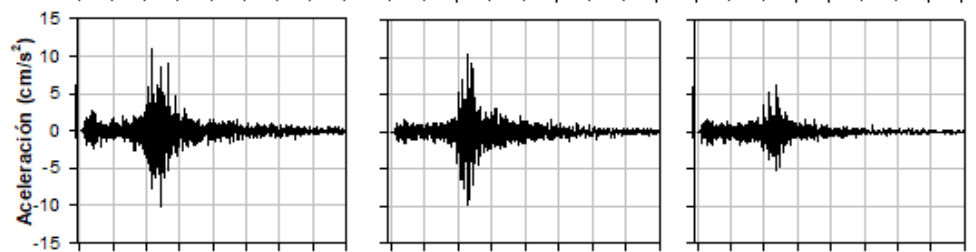

c)
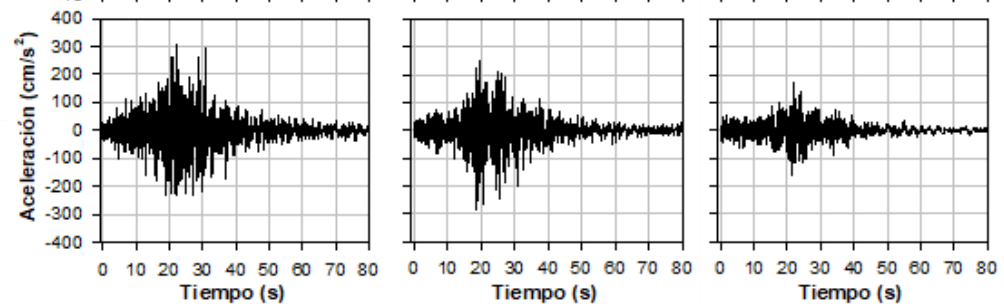

Figura 12. Acelerogramas sintéticos de las tres componentes ortogonales: ahmax, ahmin y avert. a) Semilla, b) Función de Green en sitio y c) Acelerograma escalado a la intensidad sísmica de interés

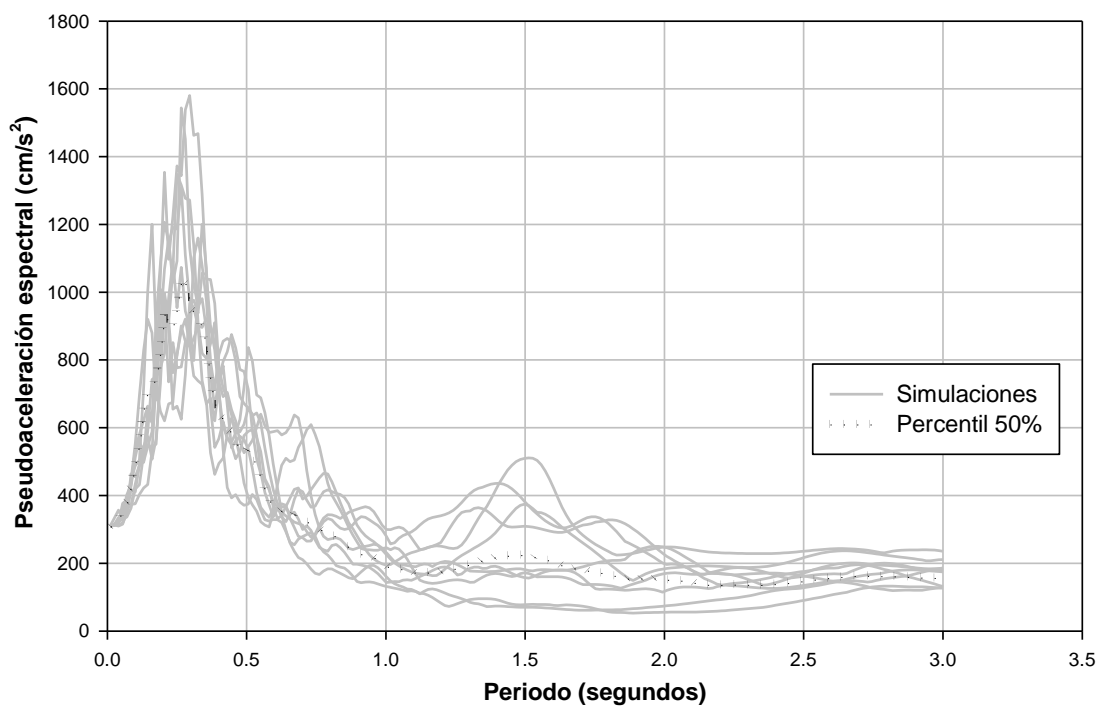

Figura 13. Espectros de respuesta elástica de 10 acelerogramas sintéticos generados con el método híbrido propuesto por Ismael y Esteva (2006), asociados a una probabilidad de excedencias del $50 \%$ en 100 años y un porcentaje de amortiguamiento del $5 \%$ con respecto al crítico

\section{Representación probabilista de las propiedades mecánicas y de resistencia de los materiales de la cortina.}

Durante el análisis estructural se consideraron 7 parámetros de forma probabilista de los que 5 corresponden a la caracterización del concreto y 2 al contacto cortina-cimentación. Todos los parámetros fueron representados por medio de funciones lognormales de probabilidad $\log [\lambda, \zeta]$. La tabla 1 resume los parámetros de las funciones lognormales utilizadas. Tanto la relación de Poisson $v$ como el ángulo de dilatancia $\psi$ del concreto se tomaron de forma determinista, con valores de 0.20 y $36^{\circ}$, respectivamente. 
También se tomaron de forma determinista las propiedades de la roca de cimentación como su densidad, módulo de elasticidad y relación de Poisson. El módulo de corte $(G)$ del contacto cortinacimentación se obtuvo conforme a la expresión $G=E_{c} / 2(1+v)$, donde $E_{c}$ es el módulo de elasticidad del concreto.

La resistencia a compresión del concreto $\left(f^{\prime} c\right)$ fue correlacionada con su resistencia a tensión $(f t)$ y su módulo de elasticidad $\left(E_{c}\right)$. Para encontrar la correlación entre las resistencias a compresión y tensión se utilizó la ecuación 15. La correlación se estableció a través de 459 pruebas de resistencia a compresión simple y tensión indirecta de una presa construida en el norte de México. Se obtuvo que $\phi_{t}$ se caracteriza mediante una función de densidad de probabilidades lognormal Log[-1.03,0.29]. Para el módulo de elasticidad se utilizó una expresión similar a la de la ecuación 15, con $\phi_{E}$ ajustada a una función $\log [8.63,0.16]$.

$$
f_{t}=\phi_{t} \sqrt{f^{\prime} c}
$$

Para cada simulación se eligió aleatoriamente un arreglo para las discretizaciones en la base de la cortina, por lo que las zonas con cohesión y fricción $(\mathrm{C}+\mathrm{F})$ y las zonas con fricción $(\mathrm{F})$ tuvieron posiciones distintas a las que se muestran en la figura 6. Lo anterior permite considerar la variación espacial de la cohesión en el contacto analizado conforme a las observaciones efectuadas por Westberg y Johansson (2013).

Tabla 1. Propiedades mecánicas y de resistencia de los materiales de la cortina

\begin{tabular}{|c|c|c|c|c|c|c|c|}
\hline Ubicación & Parámetro & Simbología & $\begin{array}{l}\text { Valor } \\
\text { medio }\end{array}$ & Unidad & $\lambda$ & $\zeta$ & Fuente de información \\
\hline \multirow{5}{*}{ Cortina } & $\begin{array}{l}\text { Densidad del } \\
\text { concreto }\end{array}$ & $\gamma_{c}$ & 2,300 & $\mathrm{~kg} / \mathrm{m}^{3}$ & 7.74 & 0.03 & Densidad típica del concreto y $\mathrm{COV}=0.03$. \\
\hline & $\begin{array}{l}\text { Resistencia a } \\
\text { compresión } \\
\text { (estática) }\end{array}$ & $f^{\prime} c$ & 12 & $\mathrm{MPa}$ & 2.47 & 0.16 & $\begin{array}{l}\text { Resistencia a compresión supuesta con } \\
\text { COV=0.16. En los análisis, } f^{\prime} c \text { se incrementó } \\
\text { un } 10 \% \text { para considerar los valores dinámicos } \\
\text { conforme a USBR (1999). }\end{array}$ \\
\hline & $\begin{array}{l}\text { Factor de } \\
\text { resistencia a } \\
\text { tensión (estática) }\end{array}$ & $\phi_{t}$ & 0.37 & Adim. & -1.03 & 0.29 & $\begin{array}{c}\text { Correlación a través de } 459 \text { pruebas. En los } \\
\text { análisis, } f t \text { se incrementó un } 40 \% \text { para } \\
\text { considerar los valores dinámicos conforme a } \\
\text { USBR (1999). }\end{array}$ \\
\hline & $\begin{array}{l}\text { Factor del módulo } \\
\text { de elasticidad. }\end{array}$ & $\phi_{E}$ & 5,650 & Adim. & 8.63 & 0.16 & $\begin{array}{l}\text { Correlación a través de } 459 \text { pruebas. No se } \\
\text { consideraron incrementos de valores estáticos a } \\
\text { dinámicos conforme a USBR (1999). }\end{array}$ \\
\hline & $\begin{array}{l}\text { Energía de } \\
\text { fractura }\end{array}$ & $G_{f}$ & 200 & $\mathrm{~N} / \mathrm{m}$ & 5.22 & 0.39 & $\begin{array}{c}\text { Tomado con base en datos de Bhattacharjee y } \\
\text { Leger (1992). }\end{array}$ \\
\hline \multirow{2}{*}{$\begin{array}{l}\text { Contacto } \\
\text { Cortina- } \\
\text { cimentación }\end{array}$} & $\begin{array}{l}\text { Coeficiente de } \\
\text { fricción }\end{array}$ & $\mu$ & 1 & Adim. & $\begin{array}{c}- \\
0.006\end{array}$ & 0.11 & COV=0.11 según Westberg (2010). \\
\hline & Cohesión & $c$ & 1.2 & $\mathrm{MPa}$ & 0.094 & 0.42 & COV=0.44 según Westberg (2010). \\
\hline
\end{tabular}

El muestreo de las propiedades mecánicas y de resistencia de la cortina se efectuó con el método del Hipercubo Latino (HL). A través de este método puede reducirse el número de simulaciones utilizadas para la estimación de la probabilidad de falla pues, a diferencia del método de Monte Carlo, el método del Hipercubo Latino (HL) involucra un esquema estratificado para mejorar la cobertura del espacio de la muestra; la estratificación se cumple dividiendo el eje vertical de la gráfica de la función de distribución de probabilidad de una variable aleatoria $X_{j}$ en $n$ intervalos de igual longitud.

\section{Probabilidad de falla por agrietamiento de la cortina. Modelo MEF2D}

La estimación de la probabilidad de falla ante el agrietamiento del cuerpo de la cortina se llevó a cabo mediante un análisis dinámico incremental (IDA), utilizando 3 grupos de 10 vectores de propiedades mecánicas y de resistencia conformados aleatoriamente con el método de muestreo del Hipercubo Latino 
(HL), utilizando las funciones lognormales de probabilidad con los parámetros presentados en la tabla 1. En total se desarrollaron 176 análisis dinámicos de los que se obtuvieron 30 valores de la intensidad de falla; 19 correspondieron a una falla por agrietamiento en la dirección aguas abajo y 11 en la dirección aguas arriba.

Los resultados de interés del modelo MEF2D son la historia de desplazamientos $\delta_{x}(t)$ en los puntos A y B, así como la fuerza cortante en la base $V_{X(t)}$ representados en la figura 14. En la figura 15 se muestra un ejemplo de los desplazamientos relativos entre los puntos A y B, calculados como la sustracción definida por $\delta_{x A}(t)-\delta_{x B}(t)$, así como entre los puntos B y $\mathrm{C}$, estimados como $\delta_{x B}(t)-\delta_{x C}(t)$, ambos en milímetros, para cada intervalo de tiempo $t$. Los desplazamientos relativos positivos son en la dirección aguas debajo de la cortina mientas que los negativos son en la dirección aguas arriba. El comportamiento estructural de la cortina puede resumirse a partir de la figura 16 y figura 17 que muestran el resultado de los 176 análisis desarrollados. En estas figuras $\Delta_{x A-x B}$ y $\Delta_{x B-x C}$ son el logaritmo natural de los desplazamientos relativos máximos encontrados en cada una de las series $\delta_{x A}(t)-\delta_{x B}(t)$ y $\delta_{x B}(t)-$ $\delta_{x C}(t)$, respectivamente. La variable $\eta$ que se muestra en las figuras 16 y 17 , representa la demanda estructural, pues está calculada como la relación entre las pseudoaceleraciones espectrales correspondientes al periodo fundamental de la cortina $\left(S_{a}\right)$ de cada simulación y la pseudoaceleración espectral media de falla $\left(S_{a \mu}\right)$ obtenida de igual manera, pero considerando los valores medios de las propiedades mecánicas y de resistencia presentados en la tabla 1.

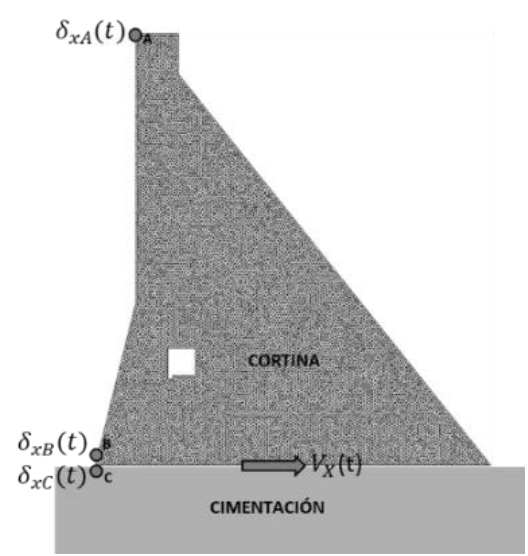

Figura 14. Resultados de interés en el modelo MEF2D para la estimación de la probabilidad de falla ante el agrietamiento de la cortina y el deslizamiento de su base

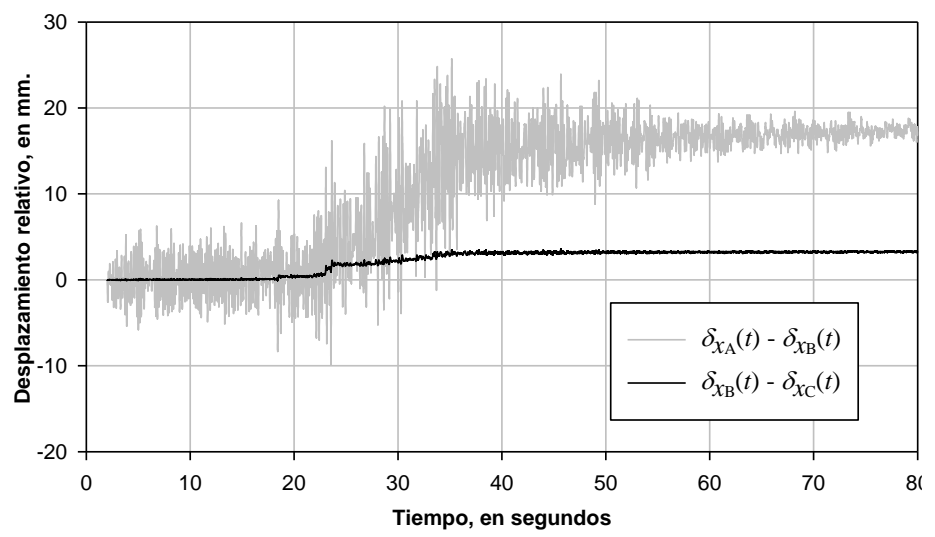

Figura 15. Ejemplo de historia de desplazamientos relativos entre los puntos A-B y B-C mostrados en la figura 14 


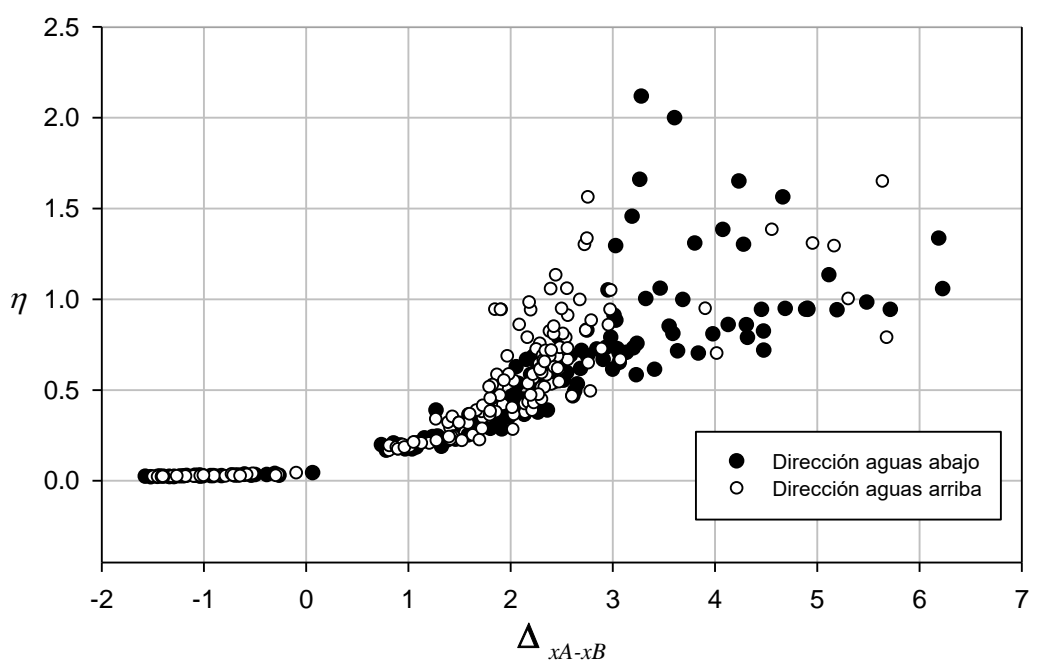

Figura 16. Resultados del desplazamiento relativo de la corona de la cortina (A) con respecto a su base (B) y la demanda sísmica $(\eta)$ para los 176 análisis desarrollados

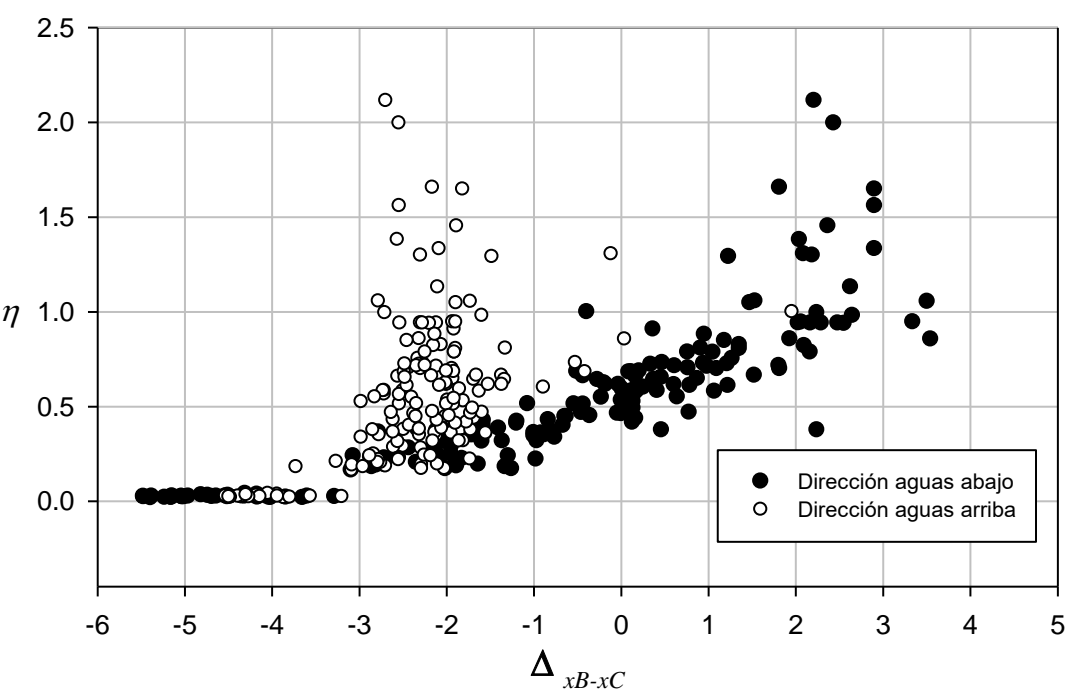

Figura 17. Resultados del desplazamiento relativo de la base de la cortina (B) con respecto a la cimentación $(\mathrm{C})$ y la demanda sísmica $(\eta)$ para los 176 análisis desarrollados

Para cada análisis se obtuvo el índice de reducción de rigidez secante expresado como $I_{R R S}=\left(K_{0}-\right.$ $K) / K_{0}$. El ejemplo de los ciclos de histéresis cuando la cortina exhibe ambos comportamientos, lineal y no lineal, se presenta en la figura 18. La rigidez inicial $K_{0}$ está calculada como el cociente entre la fuerza cortante y el desplazamiento histerético máximo asociado, cuando la cortina exhibe un comportamiento lineal, ante intensidades sísmicas bajas. La rigidez secante $K$ está calculada de la misma forma, pero cuando la cortina exhibe un comportamiento no lineal ante intensidades sísmicas significativas y los desplazamientos han producido grietas. Para diferenciar las rigideces de la cortina en las direcciones aguas abajo y aguas arriba se incluyó un signo + y - como subíndice, respectivamente. Nótese en el ejemplo de la figura 18 que la cortina exhibe un comportamiento no lineal más significativo en el dirección aguas abajo, por lo que las grietas están formadas en el paramento aguas arriba, mientras que en la dirección aguas arriba la rigidez secante $K_{(-)}$es muy parecida a la rigidez secante $K_{0(-)}$, exhibiendo un comportamiento lineal en 
esta dirección. Los valores del índice $I_{R R S}$ calculados para los 176 análisis dinámicos se muestran en la figura 19.

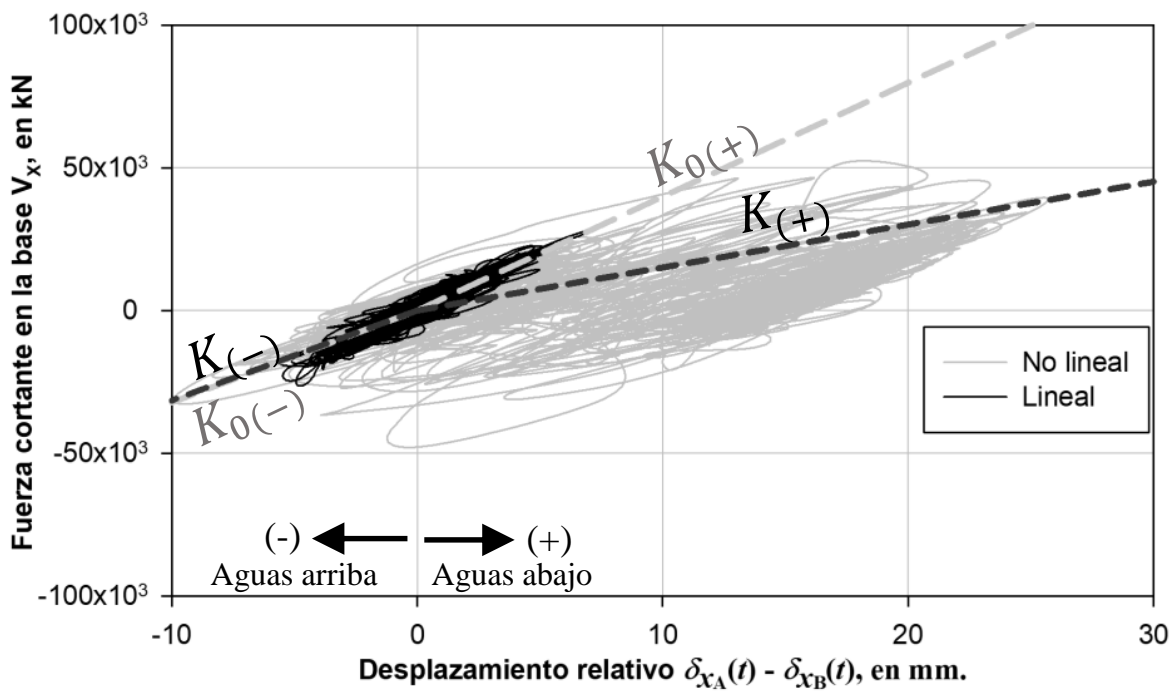

Figura 18. Ejemplo de dos ciclos de histéresis. Uno exhibiendo comportamiento lineal y otro exhibiendo comportamiento no lineal en el cuerpo de la cortina

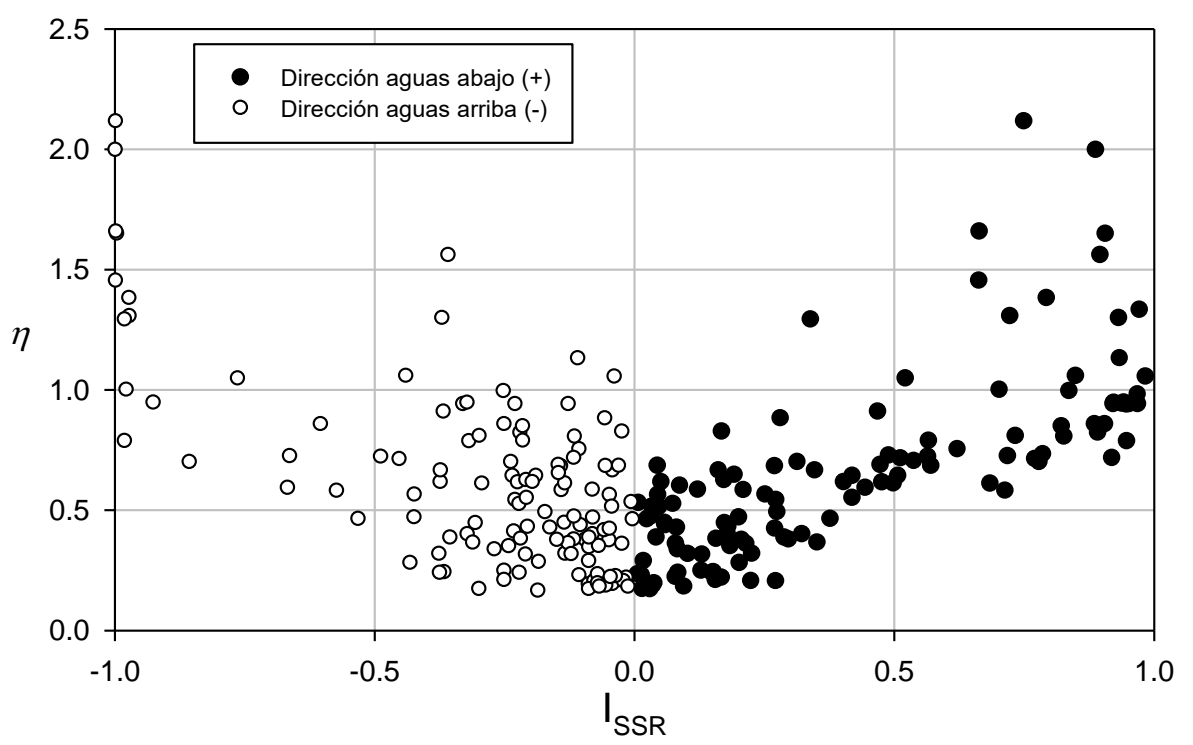

Figura 19. Valores del índice de reducción de rigidez secante $I_{S S R}$ para las direcciones aguas arriba (+) y aguas abajo (-) de la cortina

Teóricamente la condición de falla se cumple cuando la rigidez secante $K=0$, lo que implica que $I_{R R S}=1$. Sin embargo, para valores de $I_{R R S}$ ligeramente menores que 1 se observaron daños considerables en el modelo MEF2D, con patrones de agrietamiento que atraviesan completamente el cuerpo de la cortina, como se muestra en la figura 20. Considerando lo anterior, se registraron las intensidades sísmicas mínimas de falla $\left(Y_{F}\right)$ que produjeron un valor de $I_{R R S}$ mayor o igual a 0.85 . 

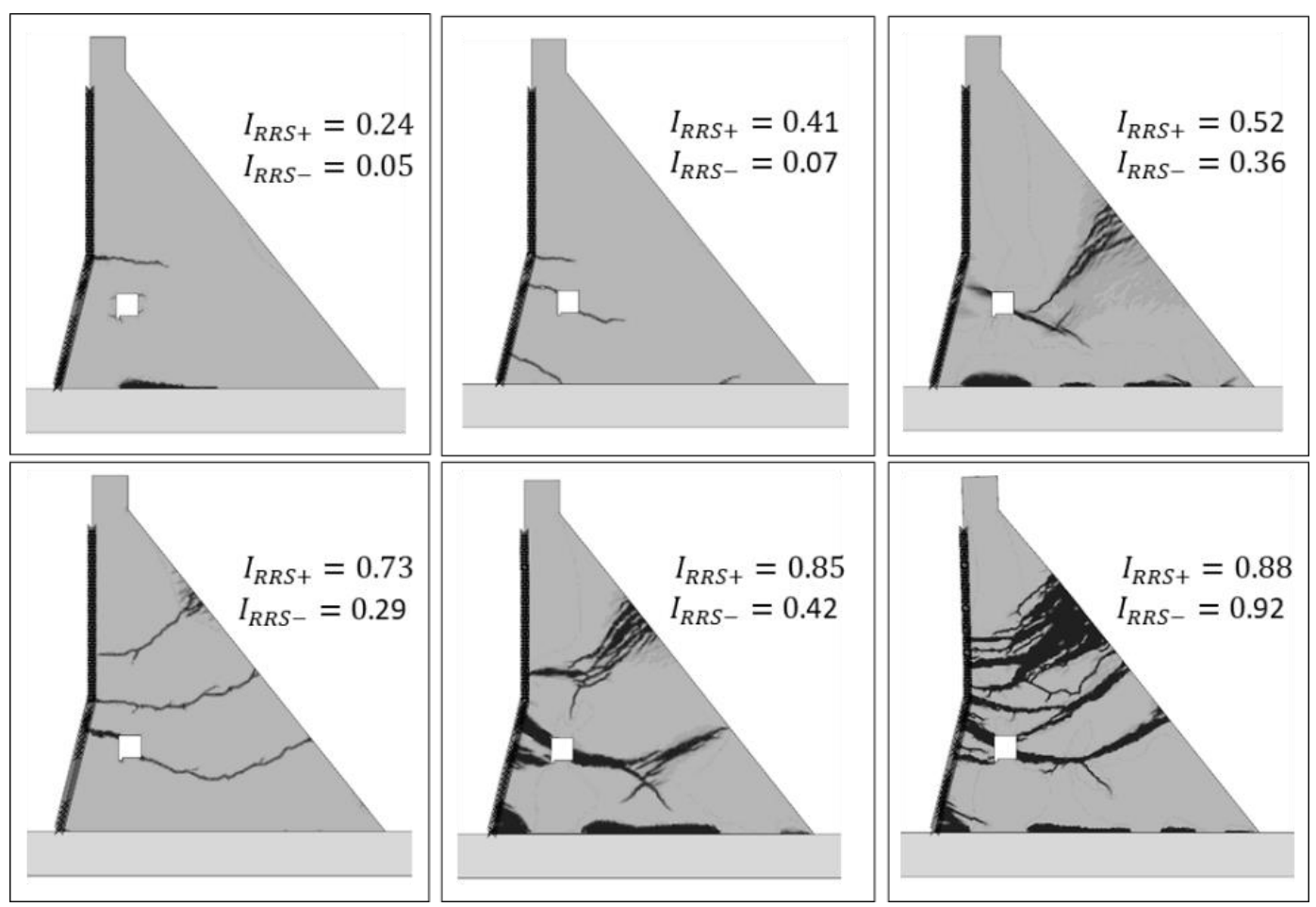

Figura 20. Ejemplo de patrones de agrietamiento y valores calculados de $I_{S S R}$ con base en los ciclos de histéresis obtenidos con el modelo MEF2D

Para la estimación del índice de confiabilidad se requiere la aplicación de la ecuación 8, para las intensidades sísmicas o parámetros de demanda que satisfacen la condición de $I_{R R S} \geq 0.85$. En la tabla 2 se presentan los parámetros $E\left(Z_{F}\right)$ y $\sigma\left(Z_{F}\right)$ obtenidos mediante un análisis de máxima verosimilitud, pero haciendo que $Y_{F}=\eta$, por lo que $Z_{F}=\ln [\eta]$. Se consideró a PGA (aceleración máxima en roca) como una medida de intensidad sísmica y $\eta$ como un parámetro de demanda. También, se evaluaron tres casos de falla, a saber:

- $\mathcal{F}_{d s}$, falla por agrietamiento en la dirección aguas abajo, utilizando únicamente los 19 valores de la intensidad sísmica de falla para esta condición.

- $\mathcal{F}_{u s}$, falla por agrietamiento en la dirección aguas arriba, utilizando únicamente los 11 valores de la intensidad sísmica de falla para esta condición.

- $\mathcal{F}_{d s}$ ó $\mathcal{F}_{u s}$, utilizando los 30 valores obtenidos de la intensidad sísmica de falla, independientemente de si el agrietamiento se presentó hacia aguas arriba o aguas abajo.

Tabla 2. Parámetros obtenidos $\left[E\left(Z_{F}\right), \sigma\left(Z_{F}\right)\right]$ para la falla por agrietamiento utilizando el modelo MEF2D

\begin{tabular}{cccc}
\hline $\begin{array}{c}\text { Medida de intensidad } \\
\text { sísmica o demanda }\end{array}$ & $\mathcal{F}_{d s}$ & \multicolumn{1}{c}{$\mathcal{F}_{u s}$} & $\mathcal{F}_{d s}$ ó $\mathcal{F}_{u s}$ \\
\hline PGA & {$[6.955,0.196]$} & {$[6.971,0.136]$} & {$[6.96,0.176]$} \\
$\eta$ & {$[-0.018,0.185]$} & {$[0.28,0.355]$} & {$[0.09,0.294]$} \\
\hline
\end{tabular}

Las funciones de confiablidad se presentan en figura 21 para los tres casos de falla descritos anteriormente. En esta figura $\beta$ es el índice de confiabilidad propuesto por Cornell en 1969 y $h$ representa el nivel fijo en el embalse considerado durante los análisis dinámicos. Finalmente, la función de probabilidad 
de falla $p_{F}(\eta \mid h)$ se presenta también en la figura 21 , calculada como $p_{F}(\eta \mid h)=\Phi(-\beta)$, donde $\Phi(\cdot)$ se refiere a la función de distribución de probabilidad normal estándar.
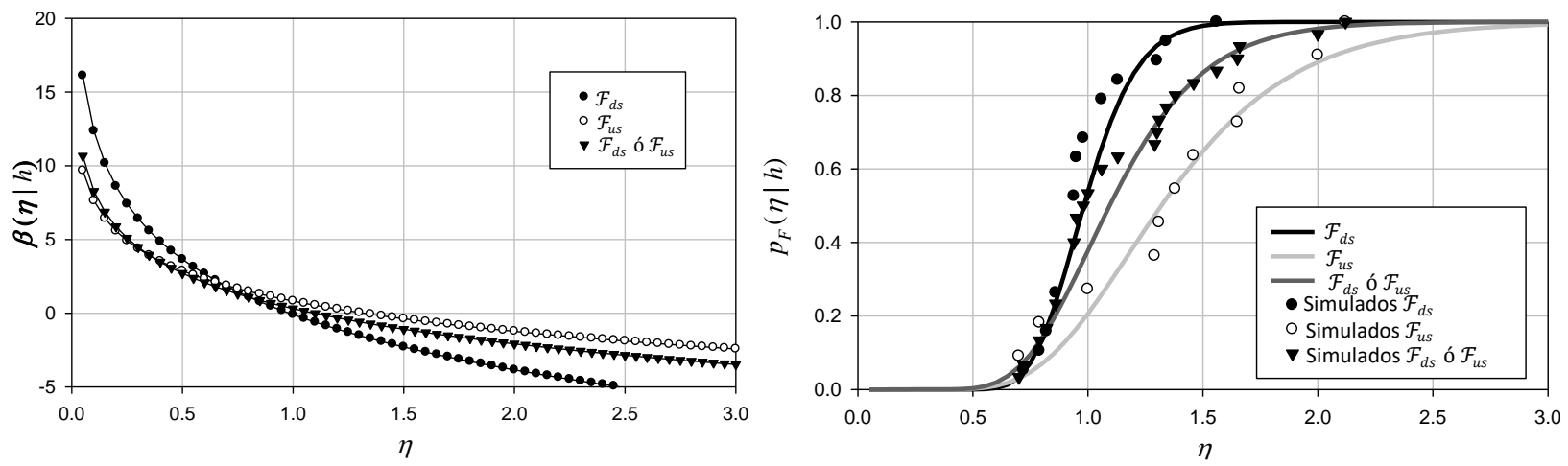

Figura 21. Funciones de confiabilidad sísmica (izquierda) y curvas de fragilidad (derecha) ante el agrietamiento de la cortina

\section{Probabilidad de falla por deslizamiento en la base de la cortina. Modelo MEF2D}

Se evaluó el deslizamiento en la base de la cortina $\left(\mathrm{S}_{l b_{-} M E F 2 D}\right)$ mediante el valor máximo de la historia en el tiempo $t$ de los desplazamientos relativos entre los puntos $\mathrm{B}$ y $\mathrm{C}$ mostrados en la figura 14. El deslizamiento puede entonces expresarse matemáticamente conforme a la ecuación 16.

$$
S_{l b \_M E F 2 D}=\max _{0 \leq t \leq 80}\left[\delta_{x B}(\mathrm{t})-\delta_{x C}(\mathrm{t})\right]
$$

La estimación de la probabilidad de falla considera que el deslizamiento ocurre exclusivamente en la dirección aguas abajo (ver figura 17). Esta suposición tiene un sentido físico al considerar que las solicitaciones en la cortina que producen el deslizamiento actúan mayormente en la dirección aguas abajo. De acuerdo con la función de estado límite presentada en la ecuación 9, la falla se alcanza cuando el deslizamiento $S_{l b_{-} M E F 2 D}$ es mayor que $150 \mathrm{~mm}$. Sin embargo, en ninguno de los 176 análisis dinámicos desarrollados se alcanzó esa condición. Por lo anterior, se procedió de la manera siguiente:

1. Se ajustaron los valores de $\eta$ en función de $\Delta_{x B-x C}$ mediante una regresión no lineal de la forma $\eta=a e^{b\left(\Delta_{x B-x C}\right)}$, obteniéndose un coeficiente de correlación $R^{2}=0.91$. Es útil recordar que: $\Delta_{x B-x C}=\ln \left[S_{l b \_M E F 2 D}\right]=\ln \left\{\max _{0 \leq t \leq 80}\left[\delta_{x B}(t)-\delta_{x C}(t)\right]\right\}$.

El resultado de la regresión se muestra en la figura 22.

2. Se obtuvo una función lognormal de probabilidad partiendo de los 176 valores de $S_{l b \_M E F 2 D}$ obtenidos con el modelo MEF2D. Los parámetros de la función de probabilidad obtenida fueron $\log [-0.77,2.32]$.

3. Con la función de probabilidad de $S_{l b_{-} M E F 2 D}$ se generaron 10,000 valores aleatorios.

4. Utilizando la regresión obtenida en el paso 1 se calcularon 10,000 valores de $\eta$, considerando que $\Delta_{x B-x C}=\ln \left[S_{l b \_M E F 2 D}\right]$ y $\eta=a e^{b\left(\Delta_{x B-x C}\right)}$.

5. Se ajustó una función lognormal de probabilidad a los valores de $\eta$ que satisficieron la condición de $S_{l b_{-} M E F 2 D}>150 \mathrm{~mm}$, obteniendo los parámetros con el método de máxima verosimilitud.

La función lognormal de probabilidad obtenida en el paso 5 proporciona los parámetros $E\left(Z_{F}\right)$ y $\sigma\left(Z_{F}\right)$ necesarios para obtener el índice de confiabilidad $\beta$ conforme a la ecuación 8 (ver tabla 3). Tanto la función de confiabilidad como la curva de fragilidad ante el deslizamiento se muestran en la figura 23. 


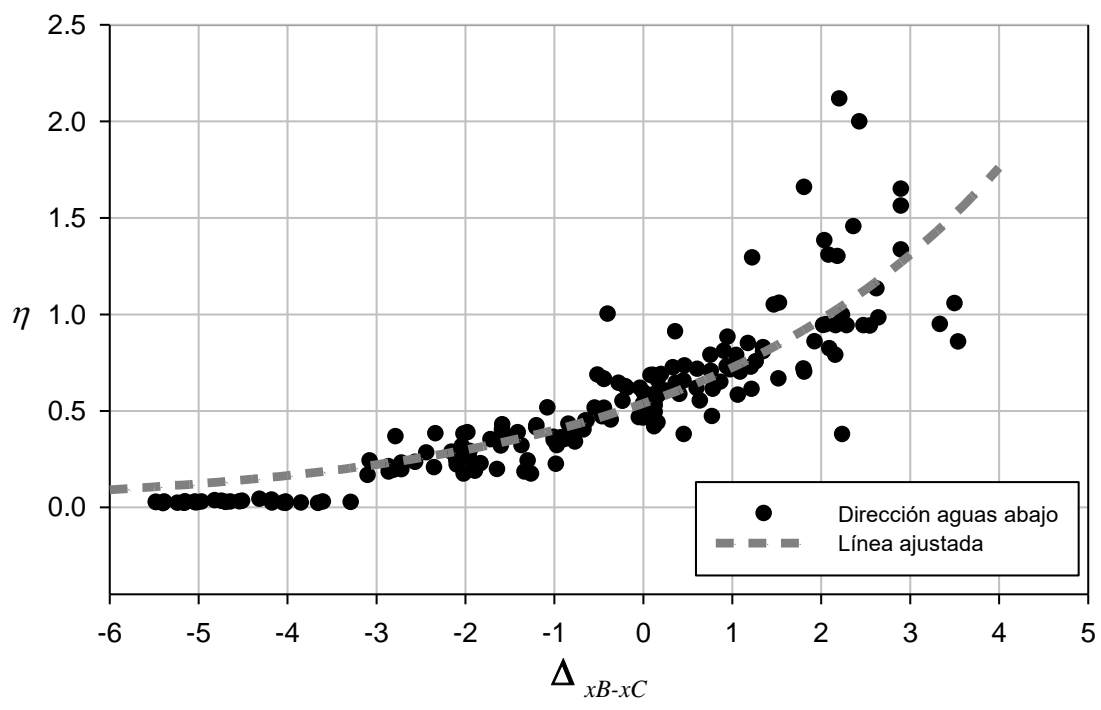

Figura 22. Resultados del ajuste de los valores de $\eta$ en función de los desplazamientos relativos máximos entre la base de la cortina y la cimentación (deslizamientos) obtenidos con el modelo MEF2D

Tabla 3. Parámetros obtenidos $\left[E\left(Z_{F}\right), \sigma\left(Z_{F}\right)\right]$ para la falla por deslizamiento utilizando el modelo MEF2D

\begin{tabular}{cc}
\hline $\begin{array}{c}\text { Medida de intensidad } \\
\text { sísmica o demanda }\end{array}$ & $S_{l b_{-} M E F 2 D}$ \\
\hline PGA & {$[7.754,0.135]$} \\
$\eta$ & {$[1.055,0.183]$} \\
\hline
\end{tabular}
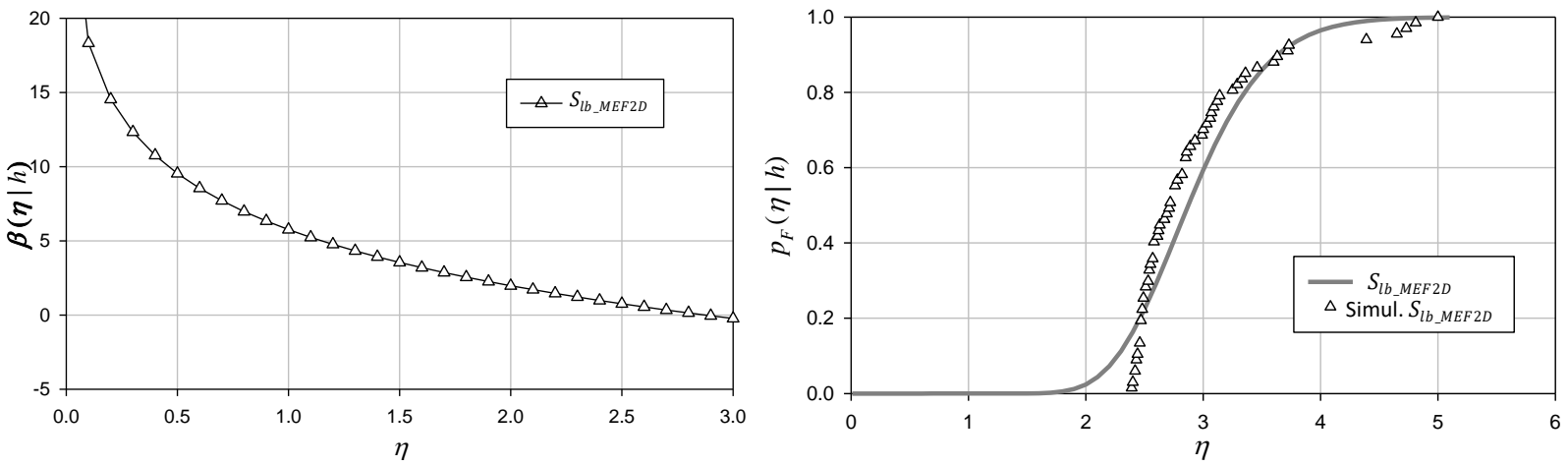

Figura 23. Funciones de confiabilidad sísmica (izquierda) y curvas de fragilidad (derecha) ante el deslizamiento en la base de la cortina utilizando el modelo MEF2D

\section{Probabilidad de falla por deslizamiento en la base de la cortina. Modelo MCR}

Si se considera que la cortina de la presa es un cuerpo rígido y existe variación espacial de la cohesión y el ángulo de fricción en el contacto de la base con la cimentación mediante $k$ discretizaciones, entonces el factor de seguridad ante el deslizamiento $(F S S)$ puede calcularse como:

$$
F S S=\frac{\sum_{i=1}^{k}\left[\mathrm{c}_{i} \mathrm{~A}_{i}+\mathrm{N}_{i} \mu_{i}\right]}{\sum F_{h}}
$$


donde $c_{i}$ es la cohesión, $A_{i}$ es el área de la discretización, $N_{i}$ es la fuerza normal actuando en la discretización, $\phi_{i}$ es el ángulo de fricción de la discretización y el término $\sum F_{h}$ indica la sumatoria de todas las fuerzas horizontales actuando en la cortina: presión hidrostática, presión de azolves, fuerza inercial horizontal, presión hidrodinámica y presión dinámica de azolves.

Tomando la ecuación 17 en un contexto probabilista, tanto $c_{i}$ como $\mu_{i}$ han sido considerados aleatorios con funciones de probabilidad lognormales definidas por los parámetros mostrados en la tabla 1. El término $A_{i}$ es determinista pues depende del tamaño asignado a las discretizaciones; en un análisis bidimensional se considera que la sección de la cortina tiene un espesor unitario, por lo que $A_{i}=L_{i}$, siendo $L_{i}$ la longitud de cada discretización. La fuerza normal resultante depende de tres factores principales: el peso propio de la cortina, el empuje vertical provocado por la subpresión y las fuerzas inerciales verticales. De estos tres factores tanto el peso propio $W$ como las fuerzas inerciales verticales $Q_{v}$ pueden ser consideradas de forma probabilista. Por un lado, $W=A_{D} \gamma_{c}$ donde $A_{D}$ es el área total de la sección transversal de la cortina y $y_{c}$ es la densidad del concreto que se caracteriza mediante una función lognormal de probabilidad conforme a la tabla 1 . Por su parte, $Q_{v}=W k_{v}$ donde $k_{v}$ es el coeficiente sísmico vertical; la caracterización probabilista de $W$ ya fue señalada anteriormente. La subpresión depende del nivel del agua y de la eficiencia del sistema de drenaje de la cortina, cuyos valores se han considerado como deterministas. También, las acciones que determinan $\sum F_{h}$ son deterministas, pues dependen exclusivamente del nivel del agua y del nivel de azolves, excepto las fuerzas inerciales horizontales $Q_{h}$ que se definen probabilísticamente de forma similar a $Q_{v}$.

Para obtener las funciones de confiabilidad en este caso se utilizó el método de Montecarlo generando un muestreo aleatorio crudo (MAC) mediante la obtención de 10,000 valores aleatorios de $c_{i}, \mu_{i}$ y $\gamma_{c}$ con los parámetros mostrados en la tabla 1. La base de la cortina fue dividida en 10 discretizaciones, de las que 6 incluyeron cohesión y fricción $(\mathrm{C}+\mathrm{F})$ y 4 únicamente fricción $(\mathrm{F})$, como se describe en la figura 6 . Por ello, fue necesario generar 6 grupos de valores aleatorios de $c_{i}$ y 10 grupos de $\mu_{i}$. Para las 10,000 simulaciones se desarrolló un análisis incremental del coeficiente sísmico horizontal $k_{h}$, hasta que el factor de seguridad FSS obtuvo un valor de 0.999 , implicando la falla por deslizamiento. Conviene recordar que $k_{h}=a_{0} /\left(1+2 a_{0}\right)$, actuando en la dirección aguas abajo, donde $a_{0}$ está definida como la aceleración máxima en roca (PGA) y $k_{v}=2 k_{h} / 3$ es el coeficiente sísmico vertical; de esta forma se obtuvieron 10,000 coeficientes sísmicos horizontales de falla denotados como $k_{h F}$.

Se definió $\eta$ como una medida de la demanda estructural, calculada mediante la relación entre cada uno de los coeficientes sísmicos horizontales de falla $k_{h F}$ y el coeficiente sísmico asociado a la falla utilizando los valores medios de las propiedades mecánicas y de resistencia de la cortina presentados en la tabla 1. Los valores de $\eta$ fueron ajustados a función lognormal de probabilidad mediante el método de máxima verosimilitud, el cual condujo a los parámetros $\log [0.856,0.371]$, que corresponden a la función de densidad de probabilidades mostrada en la figura 24.

Los parámetros necesarios para aplicar la ecuación 8 y obtener las funciones de confiabilidad se presentan en la tabla 4. Se ha incluido un escenario adicional donde se ignora la cohesión en la zona de contacto de la base de la cortina con la cimentación pues algunas recomendaciones de diseño especifican una revisión adicional bajo estas condiciones (MOC, 2015). Tanto la función de confiabilidad como la curva de fragilidad por el deslizamiento de la cortina en su base se presentan en la figura 25. 


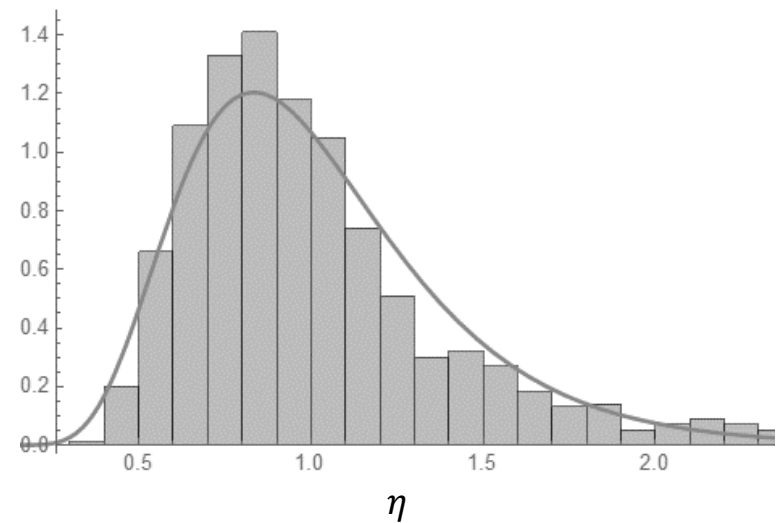

Figura 24. Función de densidad de probabilidad de $\eta$

Tabla 4. Parámetros obtenidos $\left[E\left(Z_{F}\right), \sigma\left(Z_{F}\right)\right]$ para la falla por deslizamiento utilizando el modelo MCR

\begin{tabular}{ccc}
\hline $\begin{array}{c}\text { Medida de intensidad } \\
\text { sísmica o demanda }\end{array}$ & $S_{l b_{-} M C R}$ & $S_{l b_{-} M C R}, c_{i}=0$ \\
\hline PGA & {$[7.905,0.371]$} & {$[6.386,0.065]$} \\
$\eta$ & {$[0.950,0.371]$} & {$[-0.570,0.065]$} \\
\hline
\end{tabular}
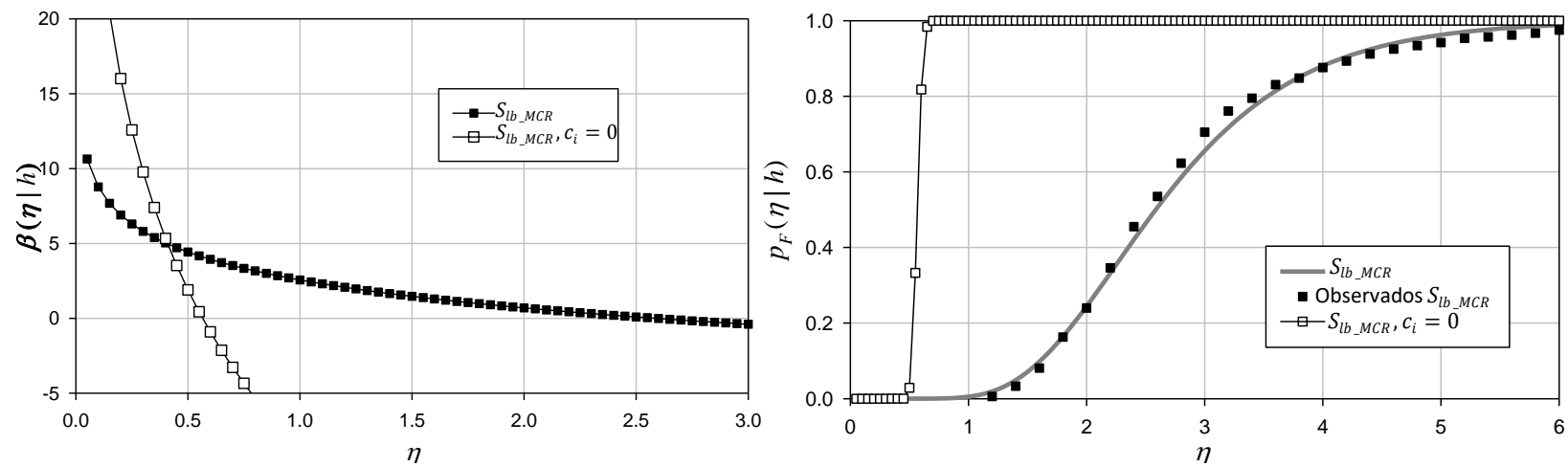

Figura 25. Funciones de confiabilidad (izquierda) y curvas de fragilidad sísmica (derecha) ante el deslizamiento en la base de la cortina utilizando el modelo MCR

\section{RESUMEN DE RESULTADOS}

Con el fin de identificar los modos de falla dominantes en la cortina se presentan las funciones de confiabilidad y las curvas de fragilidad considerando dos medidas de intensidad: $\eta$ y PGA. Aunque es reconocido que la pseudoaceleración espectral proporciona mayor precisión en los ajustes de los resultados de los análisis dinámicos, se ha utilizado PGA para efectuar la comparación de los datos obtenidos en el modelo MEF2D con los del MCR. Las funciones de confiabilidad sísmica se muestran en las figuras $26 \mathrm{y}$ 27 mientras que las curvas de fragilidad se muestran en las figuras 28 y 29.

Las funciones de confiabilidad sísmica y las curvas de fragilidad mostradas en las figuras siguientes muestran que, mientras se ignore la cohesión en el contacto de la cortina con la cimentación, la falla por deslizamiento ocurre antes de que se presente el agrietamiento de la cortina. Sin embargo, si se considera la cohesión, entonces predomina la falla por agrietamiento. Los valores de cohesión utilizados tanto en el 
modelo FEM2D como en el modelo MCR se aproximan a un valor del 10\% de la resistencia a compresión del concreto, que es un valor usual utilizado en el análisis y diseño de presas de concreto.

En relación con el agrietamiento, los resultados sugieren que la falla en dirección aguas abajo es más probable que la falla en la dirección opuesta (aguas arriba). De hecho, de las 30 intensidades sísmicas de falla obtenidas con el análisis IDA, 19 (63\%) correspondieron a una falla en la dirección aguas abajo mientras que $11(37 \%)$ correspondieron a la dirección agua arriba. Los patrones de agrietamiento obtenidos con el modelo MEF2D iniciaron principalmente en las discontinuidades geométricas de la sección transversal, como se ha reportado en la literatura.

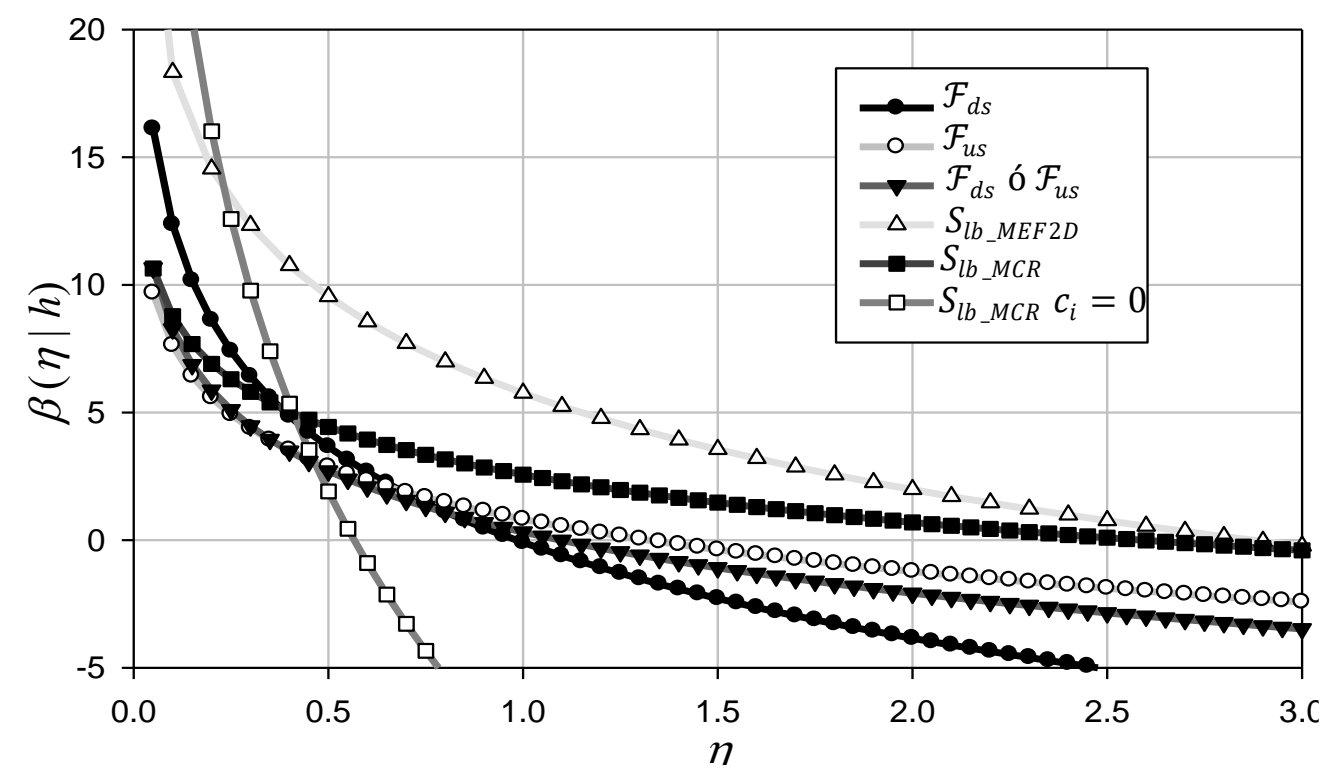

Figura 26. Funciones de confiabilidad sísmica para el agrietamiento de la cortina y el deslizamiento de su base en función de $\eta$ dado un nivel fijo $h$ en el embalse

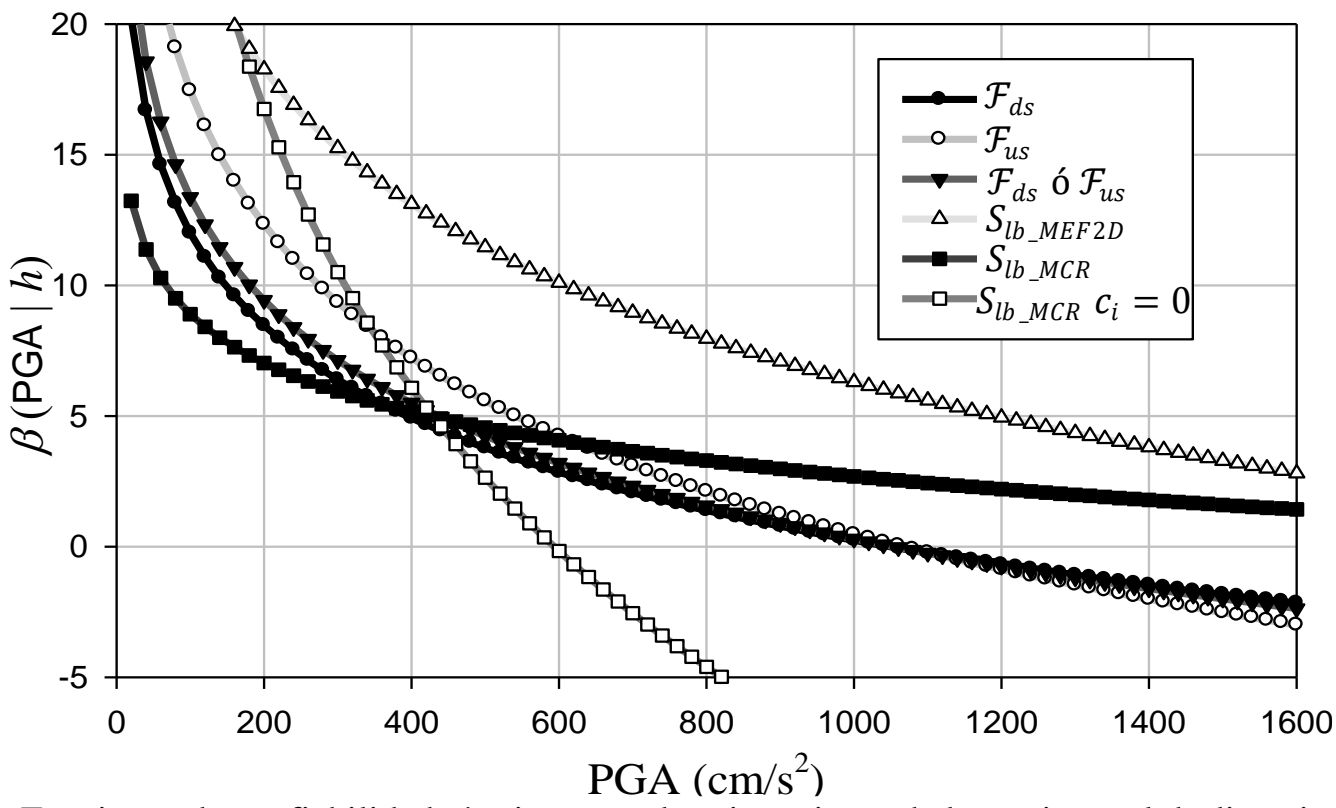

Figura 27. Funciones de confiabilidad sísmica para el agrietamiento de la cortina y el deslizamiento de su base en función de PGA dado un nivel fijo $h$ en el embalse 


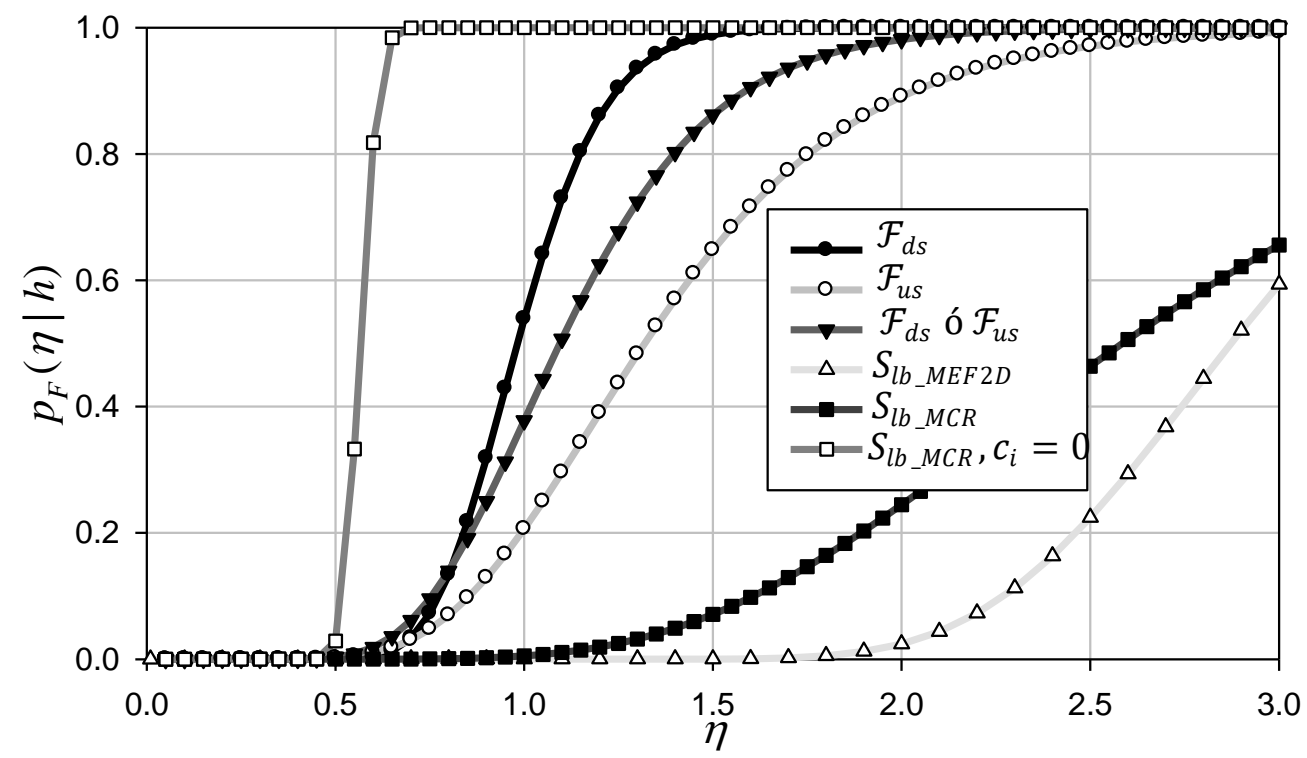

Figura 28. Curvas de fragilidad sísmica para el agrietamiento de la cortina y el deslizamiento de su base en función de $\eta$ dado un nivel fijo $h$ en el embalse

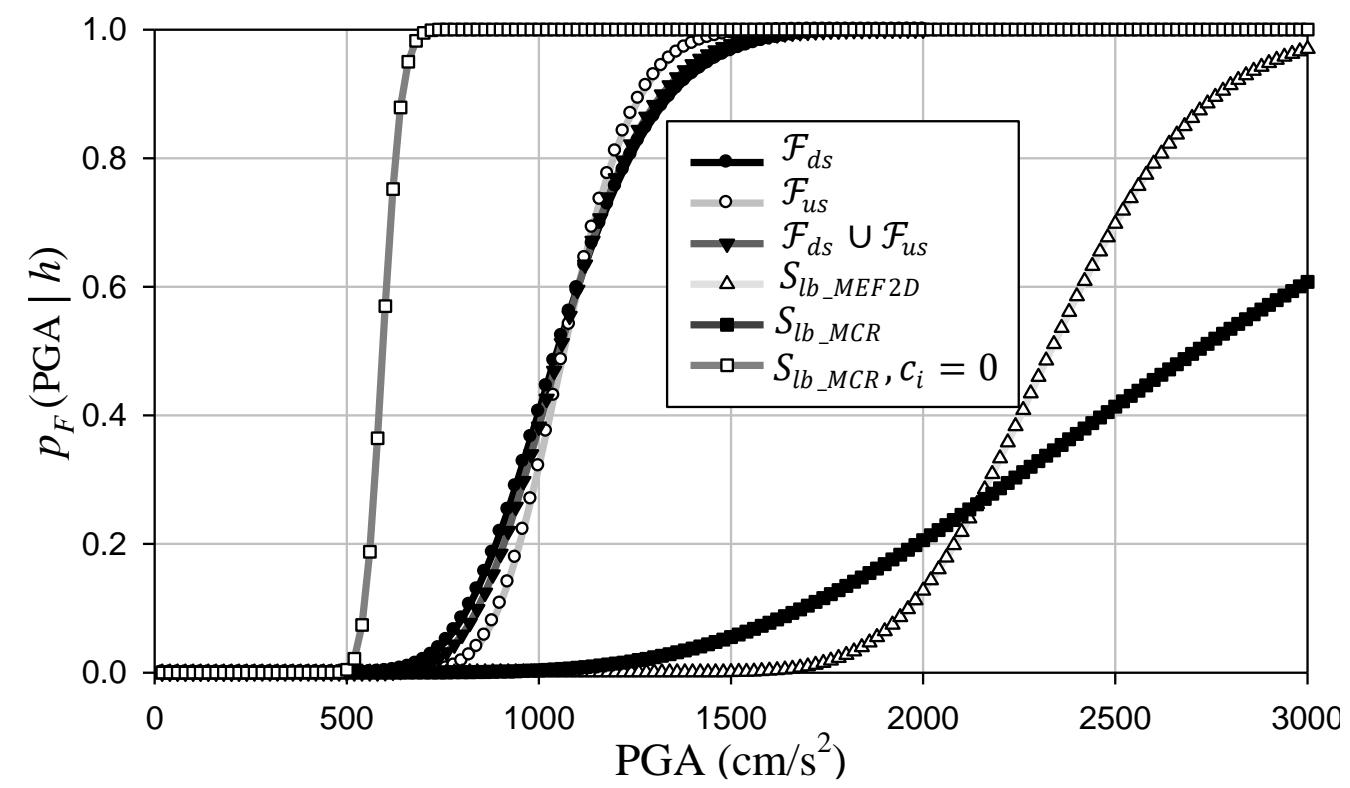

Figura 29. Curvas de fragilidad sísmica para el agrietamiento de la cortina y el deslizamiento de su base en función de PGA dado un nivel fijo $h$ en el embalse

Durante la evaluación del deslizamiento en la base de la cortina, a partir del modelo MEF2D, se observó que en ninguno de los 176 análisis desarrollados se alcanzó un deslizamiento de $150 \mathrm{~mm}$ que es la condición de falla sugerido por Bernier et al. (2016). Con los resultados del modelo MCR se observa que la probabilidad de falla por deslizamiento es notoriamente mayor si se considera únicamente la fricción que cuando se consideró la aportación de la cohesión a la resistencia del contacto. 


\section{CONCLUSIONES}

En este trabajo se presentó el planteamiento general del proceso de optimización del diseño de estrategias de seguridad, reparación o rehabilitación dentro del ciclo de vida de presas construidas con cualquier material, aunque se hace especial énfasis en presas rígidas de concreto. Bajo un enfoque económico, la estrategia que permita maximizar la función de utilidad es la más conveniente; sin embargo, la conversión de la pérdida de vidas humanas en un valor monetario es causa de controversia. Por lo anterior, la maximización de las utilidades $U_{t E}$ debe condicionarse a que la probabilidad anual de falla sea menor que la especificada en la norma mexicana NMX-AA-175-SCFI-2015.

Se mostró el proceso para la generación de acelerogramas sintéticos, incluyendo tres direcciones ortogonales, asociados a distintos niveles de intensidad sísmica $y$. Para lograr lo anterior, se aplicó el método híbrido desarrollado por Ismael y Esteva (2006) a través del procesamiento de 455 registros sísmicos de 7 estaciones ubicadas en el estado de Chiapas, México, que ocurrieron entre los años 1976 a 2016. Con la aplicación de este método fue posible escalar los registros sísmicos en función de la intensidad sísmica y en el contenido de frecuencias.

Se establecieron las funciones de estado límite para evaluar la falla de la cortina ante el agrietamiento del cuerpo y el deslizamiento de su base mediante dos modelos de comportamiento estructural: elemento finito bidimensional (MEF2D) y cuerpo rígido (MCR).

Se propone que, para el agrietamiento de la cortina, las funciones de confiabilidad se calculen aplicando el concepto del índice de reducción de rigidez secante presentado por Esteva et al. (2011) considerando las historias de desplazamientos relativos en la corona de la cortina y las fuerzas cortantes basales obtenidos mediante un modelo de elementos finitos.

Las funciones de confiabilidad sísmica y las curvas de fragilidad mostraron que, mientras se ignore la cohesión en el contacto de la cortina con la cimentación, la falla por deslizamiento ocurre antes de que se presente el agrietamiento de la cortina. Sin embargo, si se considera la cohesión, entonces predomina la falla por agrietamiento. Los resultados también sugirieron que la falla en dirección aguas abajo es más probable que la falla en la dirección opuesta (aguas arriba). De hecho, de las 30 intensidades sísmicas de falla obtenidas con el análisis IDA, 19 (63\%) correspondieron a una falla en la dirección aguas abajo mientras que $11(37 \%)$ correspondieron a la dirección agua arriba. Los patrones de agrietamiento obtenidos con el modelo MEF2D iniciaron principalmente en las discontinuidades geométricas de la sección transversal, como se ha reportado en la literatura.

La posibilidad del deslizamiento fue evaluada a través de los resultados de los modelos de elemento finito (MEF2D) considerando la variación espacial de la cohesión y el ángulo de fricción en la zona de contacto de la base de la cortina con la cimentación. La función de estado límite ante esta condición de falla está basada en Bernier et al. (2016) quienes señalaron que un deslizamiento de $150 \mathrm{~mm}$ implicaría un nivel de daño severo que causaría eventualmente el colapso de la cortina y la liberación descontrolada del agua de la presa. No obstante, en ninguno de los 176 análisis desarrollados se alcanzó esta condición, pues se produjo la falla total por agrietamiento antes de alcanzar el nivel de deslizamiento que produce la falla; en efecto, el valor mayor de deslizamiento fue de $34.82 \mathrm{~mm}$. Se anuncia que es necesario comprobar esta situación para otras alturas de cortina y configuraciones geométricas distintas de la presa. Sin embargo, cuando se efectuó un escenario ignorando la cohesión mediante un Modelo de Cuerpo Rígido (MCR) la probabilidad de falla ante el deslizamiento superó con creces a la del agrietamiento, por tanto, la cohesión tiene un impacto significativo en la probabilidad de falla ante el deslizamiento. La magnitud de la cohesión utilizada en este estudio es aproximadamente el $10 \%$ de la resistencia a compresión del concreto, que es un valor adoptado comúnmente en la práctica profesional durante el diseño de presas. 
Otro aspecto importante sobre el deslizamiento es que se ha considerado únicamente la posibilidad de que suceda en la base de la cortina; sin embargo, si en algún sitio del subsuelo se encuentran discontinuidades geológicas con resistencias bajas ante esfuerzos cortante podrían regir el comportamiento del sistema estructural, al presentarse el deslizamiento en estas zonas en lugar de la base de la cortina.

Los resultados de este estudio se basan en una única altura de cortina y en un nivel fijo del embalse, por lo que deben efectuarse estudios adicionales que permitan mostrar la influencia de estos dos parámetros en las funciones de confiabilidad sísmica.

Por otro lado, se sabe que los efectos tridimensionales en zonas topográficas angostas pueden ser importantes en el desempeño estructural de una cortina de concreto, por lo que también deben efectuarse estudios posteriores para analizar el impacto de esos efectos en las funciones de confiabilidad y la probabilidad de falla ante la amenaza sísmica.

\section{AGRADECIMIENTOS}

Este trabajo ha sido desarrollado gracias al apoyo otorgado por el Consejo Nacional de Ciencia y Tecnología (CONACYT) en forma de beca doctoral. Se hace un agradecimiento especial a los comentarios y sugerencias durante las evaluaciones semestrales y de candidatura de la Dra. Sonia Ruíz Gómez, el Dr. Juan José Pérez Gavilán-Escalante, el Dr. Carlos Javier Mendoza Escobedo y el Dr. Francisco Javier Sánchez Sesma del Instituto de Ingeniería de la UNAM. Se agradecen las licencias académicas y estudiantiles de Abaqus FEA by SIMULIA, Wolfram Mathematica y Microsoft Office.

\section{REFERENCIAS}

Aguirre, J y K Irikura (2004). "Source characterization of Mexican subduction earthquakes for the prediction of strong ground motions". 13th World Conference on Earthquake Engineering, 1 August. Issue 1572. https://www.iitk.ac.in/nicee/wcee/article/13 1572.pdf

Alamilla, J, L Esteva, J García-Pérez y O Díaz-López (2001). Earthquake engineering challenges and trends. Honoring Luis Esteva. Simulating earthquake ground motion at a site, for given intensity and uncertain source location. Instituto de Ingeniería de la UNAM. Universidad Nacional Autónoma de México. ISBN: 970-32-3699-5. DOI: 10.29104/phi-aqualac/2010-v2-2-03

Bernier, C, J Padgett, J Proulx y P Paultre (2016). "Seismic fragility of concrete gravity dams with spatial variation of angle of friction: Case Study". Journal of Structural Engineering, pp. 1-11. ISSN 07339445. DOI: 10.1061/(ASCE)ST.1943-541X.0001441

Bhattacharjee, S y P Leger (1992). "Concrete constitutive models for nonlinear seismic analysis of gravity dams - State of the art". Canadian Journal of Civil Engineering, Volumen 19, pp. 492-509. DOI: $\underline{10.1139 / 192-059}$

Bretas, E M, J V Lemos y P B Lourenco (2016). "Seismic analysis of masonry gravity dams using the discrete element method: Implementation and application". Journal of Earthquake Engineering, 20:157-184, 2016. ISSN: 1363-2469. DOI: 10.1080/13632469.2015.1085463

Burman, A (2012). "Transient analysis of aged concrete dam-foundation coupled system". Ph.D. Dissertation. Indian Institute of Technology Guwahati. http://gyan.iitg.ernet.in/bitstream/handle/123456789/230 
CONAGUA (2001). "Manual para capacitación en seguridad de presas, Módulo: Evaluación de la estabilidad en presas de concreto. Traducción autorizada de United States Bureau of Reclamation", Subdirección General Técnica, Gerencia de Ingeniería Básica y Normas Técnicas, México. Comisión Nacional del Agua. Secretaría de Medio Ambiente y Recursos Naturales.

Cornell, C A (1969). "A probability-based structural code". Journal of the American Concrete Institute, 12(66), pp. 974-985. https://www.concrete.org/publications/7446

DOF, (2016). "NMX-AA-175-SCFI-2015. Operación segura de presas parte 1.- Análisis de riesgo y clasificación de presas". Diario Oficial de la Federación-Secretaría de Economía. https://www.gob.mx/cms/uploads/attachment/file/166836

Esteva, L (1970). "Regionalización sísmica de México para fines de ingeniería”. México, Series del Instituto de Ingeniería de la UNAM. Universidad Nacional Autónoma de México. https://aplicaciones.iingen.unam.mx/ConsultasSPII/DetallePublicacion.aspx?id=152

Esteva, L, D Campos y O Diaz-López (2011). "Life-cycle optimisation in earthquake engineering". Structure and Infrastructure Engineering: Maintenance, Management, Life-Cycle Design and Performance. Volumen 7:1-2, pp. 33-49. DOI: 10.1080/15732471003588270

Esteva, L, O Díaz-López, J García-Pérez, G Sierra y E Ismael (2002). "Life-cycle optimization in the establishment of performance-acceptance parameters for seismic design". Structural Safety, Issue 24, pp. 187-204. DOI: 10.1016/S0167-4730(02)00024-3

Gogoi, I y D Maity (2007). "Influence of sediment layers on dynamic behavior". Journal of Engineering Mechanics. Volumen 133, Issue 4. DOI: 10.1061/(ASCE)0733-9399(2007)133:4(400)

ICOLD (1995). "Dam failures statistical analysis". International Comission on Large Dams. Boletín 99. https://www.icold-cigb.org/GB/publications/bulletins.asp

ICOLD (2016). "Selecting seismic parameters for large dams". International Comission on Large Dams. Boletín 148. https://www.icold-cigb.org/GB/publications/bulletins.asp

Ismael, E y L Esteva (2006). "A hybrid method for simulating strong ground motions records". First European Conference on Earthquake Engineering and Seismology, Issue 1265. Ginebra, Suiza. ISBN: 9781615676750. http://www.proceedings.com/06775.html

Jaimes, M A, A Ramírez-Gaytan y E Reinoso (2015). "Ground-motion prediction model from intermediatedepth intraslab earthquakes at the hill and lake-bed zones of Mexico City". Journal of Earthquake Engineering, 00:1-19, 2015. DOI: 10.1080/13632469.2015.1025926

Membrilla, M, I Escuder, J González y L Altarejos (2005). “Aplicación del análisis de riesgos a la seguridad de presas". Editorial UPV-Universidad Politécnica de Valencia. Ref.: 2005.2522. DOI: $\underline{10.4995 / \text { thesis/10251/7350 }}$

MOC (2008). "Manual de diseño de obras civiles. Diseño por sismo. México". Instituto de Investigaciones Eléctricas. Comisión Federal de Electricidad.

MOC (2015). "Manual de diseño de obras civiles. Diseño por sismo. México". Instituto de Investigaciones Eléctricas. Comisión Federal de Electricidad.

Ordaz, M, J Arboleda y S K Singh (1995). "A scheme of random summation of an empirical Green's Function to estimate ground motions from future large earthquake". Bulletin of the Seismological Society of America, 85(6), pp. 1635-1647. DOI: 10.1785/BSSA0850061635

Rosenblueth, E (1970). "Confiabilidad y utilidad en ingeniería". Serie azul del Instituto de Ingeniería de la UNAM. Universidad Nacional Autónoma de México. p. 48.

https://aplicaciones.iingen.unam.mx/ConsultasSPII/DetallePublicacion.aspx?id=147 
SPANCOLD (2012). "Guía técnica de explotación de presas y embalses. Tomo 1. Análisis de riesgos aplicado a la gestión de seguridad de presas y embalses". Comité Nacional Español de Grandes Presas. https: https://www.spancold.org/producto/guia-tecnica

USACE (1995). “Gravity dam design. EM 1110-2-2200”. Department of the Army. United State Army Corps of Engineers. Washintong, DC, 20314-1000. https://www.publications.usace.army.mil/EM_1110-2-2200

USACE (2001). Seismic testing of a 1/20 scale model of Koyna Dam- ERDCTR-01-17. Engineer Research and Development Center. United State Army Corps of Engineers. https://erdclibrary.erdc.dren.mil/jspui/handle/11681/8535

USACE (2014). "Safety of dams - Policy and procedures-ER 1110-2-1156". Department of the Army. United State Army Corps of Engineers. , Washintong, DC, 20314-1000. https://www.publications.usace.army.mil/ER_1110-2-1156

USBR (1976). "Design of gravity dams". United State Bureau of Reclamation. Denver, Colorado. https://www.usbr.gov/tsc/techreferences/mands/mands-pdfs/GravityDams.pdf

USBR (1999). "Dynamic properties of mass concrete obtained from dam cores". United State Department of The Interior. Bureau of Reclamation. Dam Safety Office. DSO-98-15. https://www.concrete.org/publications/internationalconcreteabstractsportal/m/details/id/4624

Vamvatsikos, D y A Cornell (2002). "Incremental dynamic analysis". Earthquake Engineering and structural dynamics. Issue 31, pp. 491-514. DOI: 10.1002/eqe.141

Westberg, M (2010). "Reliability-based assessment of concrete dam stability". Ph. D. Dissertation. Stockholm, Sweden: Lund University, Division of Structural Engineering. http: https://portal.research.lu.se/portal/en/publications/reliabilitybased-assessment-of-concrete-damstability(58629968-65db-4e4d-bac8-d66bd1cbfd67)/bibtex.html

Westberg, M y F Johansson (2013). "System reliability of concrete dams with respect to foundation stability: Application to a spillway". Journal of geotechnical and geoenvironmental engineering, American Society of Civil Engineer, 139(2), pp. 308-319. ISSN1090-0241/2013/2-308-319. DOI: $\underline{10.1061 /(A S C E) G T .1943-5606.0000761 ~}$ 